\title{
The Role of Minocycline in the Treatment of Nosocomial Infections Caused by Multidrug, Extensively Drug and Pandrug Resistant Acinetobacter baumannii: A Systematic Review of Clinical Evidence
}

\author{
Paraskevi C. Fragkou 1,*(1), Garyfallia Poulakou ${ }^{2}$, Andromachi Blizou ${ }^{1}$, Myrto Blizou ${ }^{1}$, \\ Vasiliki Rapti ${ }^{1}$, Drosos E. Karageorgopoulos ${ }^{1}$ (D), Despoina Koulenti ${ }^{3,4}$, \\ Antonios Papadopoulos ${ }^{1}$, Dimitrios K. Matthaiou ${ }^{3}$ and Sotirios Tsiodras ${ }^{1, *(D)}$ \\ 1 4th Department of Internal Medicine, Attikon University Hospital, National and Kapodistrian University of \\ Athens; 12462 Athens, Greece; mahiblizou@hotmail.com (A.B.); myrto_bl@hotmail.com (M.B.); \\ vassiarapti@gmail.com (V.R.); drkarag@gmail.com (D.E.K.); antpapa1@otenet.gr (A.P.) \\ 2 3rd Department of Medicine, Sotiria General Hospital, National and Kapodistrian University of Athens, \\ 11527 Athens, Greece; gpoulakou@gmail.com \\ 3 Adult Critical Care Unit, Attikon University Hospital, National and Kapodistrian University of Athens, \\ 12462 Athens, Greece; d.koulenti@uq.edu.au (D.K.); d.matthaiou@gmail.com (D.K.M.) \\ 4 BTCCRC, UQCCR, Faculty of Medicine, University of Queensland, Brisbane, QLD 4072, Australia \\ * Correspondence: evita.fragou@gmail.com (P.C.F); sotirios.tsiodras@gmail.com (S.T.); \\ Tel.: +30-210-583-1990 (P.C.F); +30-210-583-1440 (S.T.)
}

Received: 11 April 2019; Accepted: 30 May 2019; Published: 1 June 2019

\begin{abstract}
Treatment options for multidrug resistant Acinetobacter baumannii strains (MDR-AB) are limited. Minocycline has been used alone or in combination in the treatment of infections associated with $\mathrm{AB}$. A systematic review of the clinical use of minocycline in nosocomial infections associated with MDR-AB was performed according to the PRISMA-P guidelines. PubMed-Medline, Scopus and Web of Science TM databases were searched from their inception until March 2019. Additional Google Scholar free searches were performed. Out of 2990 articles, 10 clinical studies ( 9 retrospective case series and 1 prospective single center trial) met the eligibility criteria. In total, 223 out of $268(83.2 \%)$ evaluated patients received a minocycline-based regimen; and 200 out of $218(91.7 \%)$ patients with available data received minocycline as part of a combination antimicrobial regimen (most frequently colistin or carbapenems). Pneumonia was the most common infection type in the 268 cases $(80.6 \%$ with $50.4 \%$ ventilator-associated pneumonia). The clinical and microbiological success rates following minocycline treatment were $72.6 \%$ and $60.2 \%$, respectively. Mortality was $20.9 \%$ among 167 patients with relevant data. In this systematic review, minocycline demonstrated promising activity against MDR-AB isolates. This review sets the ground for further studies exploring the role of minocycline in the treatment of MDR-AB associated infections.
\end{abstract}

Keywords: minocycline; nosocomial infections; Acinetobacter baumannii; antimicrobial drug resistance; MDR; XDR; PDR

\section{Introduction}

Minocycline, semi-synthetic derivative of tetracycline, initially introduced in the 1960s, is a broad-spectrum antibacterial agent with activity against both aerobic and anaerobic gram-positive and gram-negative microorganisms [1-3]. Its antimicrobial properties are a result of protein synthesis 
inhibition and thus it exhibits a bacteriostatic effect. When administered orally, it is readily bioavailable as it is rapidly absorbed by the gastrointestinal tract (95-100\%) [2-5]. These properties make minocycline a potential candidate for switching from an intravenous to an oral formulation without compromising its effectiveness, which may reduce the length and the cost of hospitalization, although studies to support this hypothesis are needed. Minocycline's longevity in clinical practice provides the advantage of a well-documented safety profile with an overall adverse event incidence rate of 13 per million per year between 1966 and 2003 [6]. However, prolonged use has been associated with hepatotoxicity, photosensitivity, irreversible skin discolouration and autoimmune phenomena, like drug-induced systemic lupus-erythematosus [2].

The era of injudicious use of antibiotics has led to a multidrug resistant (MDR) microbial clone selection, especially in the healthcare settings, thus leaving limited therapeutic options for nosocomial infections. The Infectious Diseases Society of America (IDSA) has voiced concerns, particularly for a group of resistant bacteria, the-so-called ESKAPE pathogens (Enterococcus faecium, Staphylococcus aureus, Klebsiella pneumoniae, Acinetobacter baumannii, Pseudomonas aeruginosa and Enterobacter species) [7-9]. Among them, Acinetobacter baumannii is a strictly aerobic gram-negative non-lactose-fermenter coccobacillus, member of the Acinetobacter calcoaceticus-baumannii complex (ACB). A broad variety of nosocomial infections are attributable to A. baumannii such as bacteremia, hospital-acquired pneumonia (HAP) and ventilator-associated pneumonia (VAP), wound and soft tissue infections (SSTIs), meningitis and urinary tract infections, occurring mainly in critically ill hosts [10-13]. Although its virulence has been a matter of debate, an association with crude mortality rates as high as 68\% has been reported for Acinetobacter spp. [14-19]. The organism's remarkable advantage of surviving for extensive periods under different environmental conditions on hospital surfaces, may underlie its ability to cause nosocomial outbreaks [20]. A. baumannii has evolved to become one of the most important multidrug resistant organisms (MDROs), with recent nosocomial clones exhibiting a resistance against many classes of antimicrobials by genomic mutations in topoisomerases and the up-regulation of the efflux pumps expression, production of oxacillinases like OXA-23, -24 and -58 , Verona-integron-encoded metallo- $\beta$-lactamases (VIM) and New-Delhi MBL (NDM-1), as well as outer membrane porin changes [21-23].

The treatment options of MDR, extensively drug resistant (XDR) and pandrug resistant (PDR) A. baumannii infections are considerably limited. Currently, the golden standard for the treatment of infections caused by A. baumannii is carbapenems due to their intrinsic activity against this pathogen [24]. Polymyxins (polymyxin E or colistin and polymyxin B) also exhibit good activity and retain low but rising resistance rates among A. baumannii isolates [24-26]. As a result of the increasing resistance and the paucity of novel antibiotics, few treatment options have been left in the clinicians' armamentarium to confront with a continuously growing healthcare issue. As novel antimicrobial agents are yet to be developed, reinstituting the use of older antimicrobials has now become a priority. The role of an old antibiotic like minocycline in the treatment of $A$. baumannii is still being explored. The favourable safety profile and the relatively low cost, make minocycline an attractive therapeutic option. Hence, a systematic review of current data is imperative in order to codify a unifying frame of practice for the use of minocycline in the treatment of MDR A. baumannii infections.

\section{Materials and Methods}

The study protocol of this systematic review was designed according to the PRISMA-P (Preferred Reporting Items for Systematic Reviews and Meta-Analyses) guidelines [27,28]. The PRISMA-P checklist is availablein Table S1.

\subsection{Inclusion Criteria}

A study of any design was considered as eligible if it fulfilled all of the following criteria: (i) it included patients aged $\geq 18$ years old, (ii) with nosocomial infections, (iii) caused by MDR and/or XDR and/or PDR A. baumannii (or Acinetobacter calcoaceticus-baumannii complex), (iv) it reported the 
utilization of minocycline, either in an oral or in intravenous formulation, alone or in combination with other antimicrobial agents, and (v) it designated the clinical and/or the microbiological success of the minocycline or the minocycline combinations either as a primary outcome or as a secondary outcome. No restrictions in terms of: site of infection, sample size, minocycline doses, route of administration, use of minocycline combinations, publication status, country or language were applied.

\subsection{Exclusion Criteria}

Studies were excluded if: (i) they included non-adult patients or included both adult and non-adult participants but without separate analysis for adult patients, (ii) they included community acquired or both nosocomial and community acquired infections, but without separate analysis for nosocomial infections, (iii) the isolates were not specified as baumannii or A. calcoaceticus-baumannii complex, (iv) the strains were not reported as MDR and/or XDR and/or PDR, (v) they did not concisely report that A. baumannii or A. calcoaceticus-baumannii complex strains were the causative organisms of the nosocomial infections and not just colonization isolates, (vi) no minocycline was administered, (vii) they were literature or systematic reviews, published conference abstracts or book chapters (viii) the full text could not be retrieved and, (ix) they were published in a language other than English, and a translation was technically impossible or unsound.

\subsection{Definitions and Assumptions}

The definitions and assumptions that were utilized in this systematic review are shown in Table 1. However, the definitions similar but not identical with the aforementioned ones used in individual studies were also acceptable.

Table 1. Definitions used in the systematic review.

\begin{tabular}{|c|c|}
\hline Terms & Definitions \\
\hline Clinical success- & Elimination of the signs \& symptoms related to the initial infection \\
\hline Clinical Cure & for which minocycline or non-minocycline based therapies are used. \\
\hline Microbiological success- & Eradication of the causative organism from the site of infection. \\
\hline \multicolumn{2}{|l|}{ Microbiological Cure } \\
\hline Adverse Events & $\begin{array}{l}\text { Onset of signs, symptoms or laboratory findings related to } \\
\text { minocycline, its combinations or non-minocycline based regimens. }\end{array}$ \\
\hline Hospital-Acquired Infection & $\begin{array}{l}\text { The date of the site-specific infection occurs on or after the 3rd } \\
\text { calendar day of admission (the day of admission is calendar day 1) [29]. }\end{array}$ \\
\hline Antimicrobial resistance classification & $\begin{array}{l}\text { MDR: non-susceptible to } \geq 1 \text { agent of } \geq 3 \text { classes of antibiotics, XDR: } \\
\text { non-susceptible to } \geq 1 \text { agent in all but } \leq 2 \text { categories, and PDR: } \\
\text { non-susceptible to all classes or agents [30]. }\end{array}$ \\
\hline Site of infection & $\begin{array}{l}\text { Site of isolation of the MDR, XDR or PDR A. baumannii (or } A \text {. } \\
\text { calcoaceticus-baumannii complex) strain in the context of a new onset } \\
\text { of relevant signs, symptoms and laboratory markers attributable to } \\
\text { infection, based on the treating clinicians' decision. }\end{array}$ \\
\hline Surgical Site Infections & Infections that occur 30 days after surgery with no implant or within \\
\hline & $\begin{array}{l}1 \text { year if an implant is placed and the infection appears to be related } \\
\text { to surgery }\end{array}$ \\
\hline
\end{tabular}

MDR: multidrug resistant, XDR: extensively drug resistant, PDR: pandrug resistant.

\subsection{Search Strategy}

PubMed-Medline, Scopus and Web of Science ${ }^{\mathrm{TM}}$ were searched from their inception until the 20th of March 2019. The search terms that were used were: "minocycline", "acinetobacter baumannii", "gram-negative" and "resistant", used in the search string as follows: ("minocycline" AND ("acinetobacter baumannii" OR "gram-negative") AND "resistant"), applied to all search fields. For the Scopus database, the search string was: ALL ("minocycline" AND ("acinetobacter baumannii" OR "gram-negative") AND "resistant")). Hand search of studies included in previously published 
systematic reviews as well as free Google Scholar searches were performed in order to identify possibly includable trials that were not identified in the initial database search.

\subsection{Outcomes \& Prioritization}

The designated outcomes of the included studies regarding nosocomial infections caused by MDR, XDR or PDR A. baumannii strains treated with minocycline were primarily the clinical success and microbiological cure as they were defined in the "Definitions" section. Secondary outcomes that were collected in patients who received minocycline, and when available in the group of the other administered antimicrobials, were: route, duration and doses of antibiotics, duration of hospitalization (in days), mortality rate, requirement for readmission to hospital (where an adequate follow-up was obtained), reported adverse events related to minocycline or minocycline combinations, and reported adverse events to other antibiotics.

\subsection{Risk of Bias in Individual Studies EConfidence in Cumulative Evidence}

Eligible clinical studies were assessed for their quality and possible bias against the STROBE (Strengthening the Reporting of Observational Studies in Epidemiology) guidelines [31].

\subsection{Data Synthesis EStatistical Analysis}

The data collected from the retrieved studies were tabulated, grouped and consequently analyzed through an integrative synthesis method. The grouping of patients was based on the administered treatment.

\section{Results}

\subsection{Search Results}

The systematic search revealed 10 clinical studies eligible for inclusion: 9 studies from databases' systematic search and 1 study from Google Scholar free search. No additional eligible studies for inclusion were identified by the manual search of previously published systematic reviews. Figure 1 shows the selection process of the included studies.

\subsection{Study Range and Characteristics}

The 10 clinical studies satisfying all the inclusion and none of the exclusion criteria are shown in Table 2. STROBE criteria for each study are demonstrated in Table A1. For the sake of brevity, each study was assigned an "Abbreviation ID" title from $A$ to $J$ which will henceforth be utilized instead of the study's first author's name and title. In general, all but two studies were published in English. Studies $G$ and $H$ were written in Chinese, so a manual translation was carried out. Six studies were undertaken in different regions of United States, one study in Argentina, while the rest of them were performed in China (studies $E, G$ and $H$ ). All studies are dated from 2005 onwards, except study $I$ which was done in 1998. No randomized controlled trials were identified. Four out of 10 studies evaluated the outcomes of patients with nosocomial infections caused by resistant $A$. baumannii strains treated with minocycline or minocycline combinations (studies $C, D, E$ and $G$ ), studies $A$ and $F$ reported outcomes from patients that received minocycline for different pathogens, including $A$. baumannii strains, whereas the remaining studies examined the clinical effects of different antimicrobial agents in A. baumannii infections. Four studies were performed in Intensive Care Unit (ICU) patients, one study included patients from both the general wards and ICU, one from a burn department, whilst the rest of them did not define the patients' origins.

In total, 286 patients were included in these studies. Among these patients, 268 (93.7\%) were diagnosed with $A$. baumannii nosocomial infections, thus representing the sample size of interest for this systematic review. These patients were treated with minocycline monotherapy, minocycline combinations or other antimicrobial agents. The remaining 18 patients were reported as primarily 
diagnosed with MRSA or Enterobacteriaceae infections. The age range of the patients with A. baumannii infections was 18-106 years old. 152 out of the221 patients with available demographic data $(68.8 \%)$ were men.

The isolated pathogens represented a variety of resistant $A$. baumannii strains, with diversity in the definitions used by the authors (Table 3). Although the majority of the definitions are not entirely in concordance with the definitions proposed by Magiorakos et al., all but 2 studies have included strains with "at least" an MDR profile. In total, carbapenem-resistant (CR), MDR, XDR and PDR A. baumannii strains were isolated from 62 (23.1\%), 78 (29.1\%), $77(28.7 \%)$ and 51 (19\%) patients respectively, according to the resistance profile given by the authors.

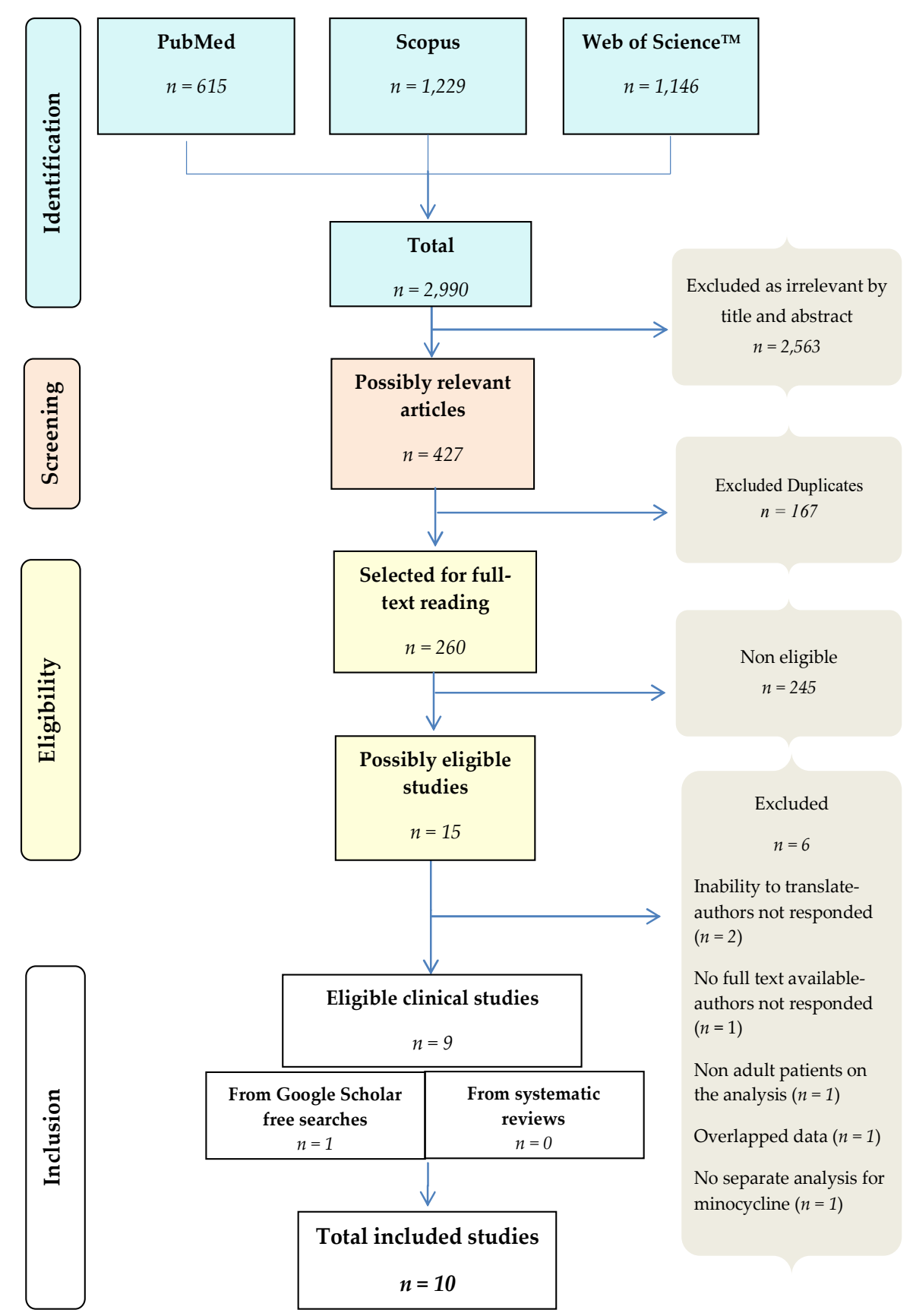

Figure 1. Systematic review flow chart. 
Table 2. Included Clinical Studies.

\begin{tabular}{|c|c|c|c|c|c|}
\hline $\begin{array}{l}\text { Abbreviation ID of } \\
\text { Study, Authors, } \\
\text { Year, Reference }\end{array}$ & $\begin{array}{l}\text { Type of Study/ Study } \\
\text { Population (N) }\end{array}$ & Country, Region & Year of Study & Setting of Study & Purpose of Study \\
\hline $\begin{array}{l}\text { Study A, Bishburg E. } \\
\text { et al., } 2014 \text { [32] }\end{array}$ & $\begin{array}{l}\text { Retrospective Case } \\
\text { Series }(\mathrm{N}=21)\end{array}$ & USA, Newark & $11 / 2009-04 / 2012$ & $\begin{array}{l}\text { Tertiary Care Hospital } \\
\text { - Ward not specified. }\end{array}$ & $\begin{array}{l}\text { To report the experience in using IV Minocycline for } \\
\text { the treatment of MRSA and resistant gram-negative } \\
\text { infections. }\end{array}$ \\
\hline $\begin{array}{l}\text { Study B, Chan J.D. et } \\
\text { al., } 2010 \text { [33] }\end{array}$ & $\begin{array}{l}\text { Retrospective Case } \\
\text { Series }(N=55)\end{array}$ & USA, Seattle & 07/2004-12/2007 & Trauma Centre - ICU. & $\begin{array}{l}\text { To describe the clinical outcomes of case series of } \\
\text { CR-AB VAP. }\end{array}$ \\
\hline $\begin{array}{l}\text { Study C, Goff D.A. } \\
\text { et al., } 2014 \text { [34] }\end{array}$ & $\begin{array}{l}\text { Retrospective Case } \\
\text { Series }(N=55)\end{array}$ & USA, Ohio & 09/2010-03/2013 & General Ward \& ICU. & $\begin{array}{l}\text { To describe an Antimicrobial Stewardship } \\
\text { Program's evaluation of Minocycline for the } \\
\text { treatment of patients with MDR-AB infections. }\end{array}$ \\
\hline Study $D$, Griffith & Retrospective Case & USA, Texas & 2005-2006 & Military Trauma & To report the outcomes of patients received \\
\hline M.E. et al.,2008 [35] & $\begin{array}{l}\text { Series (Retrospective } \\
\text { Chart Review) }(\mathrm{N}=8)\end{array}$ & & & $\begin{array}{l}\text { Centre - Ward not } \\
\text { specified. }\end{array}$ & $\begin{array}{l}\text { Minocycline as therapy for MDR-ABC traumatic } \\
\text { wound infections. }\end{array}$ \\
\hline $\begin{array}{l}\text { Study E, Ning F. et } \\
\text { al., } 2014 \text { [36] }\end{array}$ & $\begin{array}{l}\text { Retrospective Case } \\
\text { Series }(N=9)\end{array}$ & CHINA Beijing & 02/2011-03/2013 & Department of Burn. & $\begin{array}{l}\text { To report outcomes of treating extensive burns with } \\
\text { PDR-AB infections with high doses of Meropenem, } \\
\text { Cefoperazone -Sulbactam and Minocycline }\end{array}$ \\
\hline $\begin{array}{l}\text { Study F, Pogue J.M. } \\
\text { et al., } 2014 \text { [37] }\end{array}$ & $\begin{array}{l}\text { Retrospective Case } \\
\text { Series }(\mathrm{N}=9)\end{array}$ & USA, Detroit & 09/2011-n/a & $\begin{array}{l}\text { Tertiary Care Hospital } \\
\text { - Ward not specified. }\end{array}$ & $\begin{array}{l}\text { To evaluate the use of IV minocycline for the } \\
\text { treatment of CR-AB \& Enterobacteriaceae. }\end{array}$ \\
\hline $\begin{array}{l}\text { Study G, Shi Y. et al., } \\
2012[38]\end{array}$ & $\begin{array}{l}\text { Prospective Single - } \\
\text { Centre Trial }(\mathrm{N}=77)\end{array}$ & CHINA, Beijing & 01/2009-12/2009 & ICU & $\begin{array}{l}\text { To explore the effects of cefoperazone/sulbactam } \\
\text { plus minocycline on XDR-AB infections in critically } \\
\text { ill patients. }\end{array}$ \\
\hline $\begin{array}{l}\text { Study H, Wang L.W. } \\
\text { et al., 2014,[39] }\end{array}$ & $\begin{array}{l}\text { Retrospective Case } \\
\text { Series }(N=42)\end{array}$ & CHINA, Beijing & 04/2009-04/2010 & $\begin{array}{l}\text { Department of } \\
\text { Respiratory } \\
\text { Medicine/ICU. }\end{array}$ & $\begin{array}{l}\text { To analyze the clinical features of PDR-AB and } \\
\text { compare the efficacy of different antibiotic } \\
\text { treatments in aged patients with PDR-AB VAP. }\end{array}$ \\
\hline $\begin{array}{l}\text { Study I, Wood G.C. } \\
\text { et al., } 2003 \text { [40] }\end{array}$ & $\begin{array}{l}\text { Retrospective Case } \\
\text { Series }(N=7)\end{array}$ & USA, Tennessee & 01/1998-12/1998 & Trauma Centre - ICU. & $\begin{array}{l}\text { To report the use of tetracyclines for the treatment of } \\
\text { MDR-AB VAP. }\end{array}$ \\
\hline $\begin{array}{l}\text { Study J, Vila A. et al., } \\
2016 \text { [41] }\end{array}$ & $\begin{array}{l}\text { Retrospective Case } \\
\text { Series }(\mathrm{N}=3)\end{array}$ & $\begin{array}{l}\text { ARGENTINA, } \\
\text { Mendoza }\end{array}$ & 2010-2012 & $\begin{array}{l}\text { Tertiary Care Hospital } \\
\text { - Ward not specified }\end{array}$ & $\begin{array}{l}\text { To report } 3 \text { cases of MDR-AB prosthetic joint } \\
\text { infections treated with debridement and tigecycline } \\
\text { ( } 2 \text { patients received oral minocycline as maintenance } \\
\text { treatment). }\end{array}$ \\
\hline
\end{tabular}

resistant, MRSA: Methicillin-resistant Staphylococcus aureus, PDR: Pandrug resistant, USA: United States of America, VAP: Ventilator-associated pneumonia, XDR: Extensively drug resistant. 
Table 3. Characteristics of included clinical studies.

\begin{tabular}{|c|c|c|c|c|c|c|c|c|c|}
\hline $\begin{array}{l}\text { Abbreviation ID } \\
\text { of study, Authors, } \\
\text { Year, Reference }\end{array}$ & $\begin{array}{l}\text { Patient } \\
\text { Demographics }\end{array}$ & $\begin{array}{l}\text { A. baumannii } \\
\text { Infections ( } N= \\
\text { Total No. of } \\
\text { Patients) }\end{array}$ & $\begin{array}{l}\text { No. of } \\
\text { Patients on } \\
\text { Minocycline } \\
\text { (Monotherapy) }\end{array}$ & $\begin{array}{l}\text { Organisms, Resistance } \\
\text { Pattern Definition \& } \\
\text { Susceptibility Testing } \\
\text { Method }\end{array}$ & $\begin{array}{l}\text { Minocycline } \\
\text { Route of } \\
\text { Administration } \\
\text { \& Doses }\end{array}$ & $\begin{array}{l}\text { Minocycline } \\
\text { Combinations }\end{array}$ & $\begin{array}{l}\text { Other Antimicrobial Agents } \\
\text { \& Doses }\end{array}$ & $\begin{array}{l}\text { Clinical Success } \\
\text { Definition }\end{array}$ & $\begin{array}{l}\text { Microbiological } \\
\text { Success Definition }\end{array}$ \\
\hline $\begin{array}{l}\text { Study A, Bishburg } \\
\text { E. et al., 2014 [32] }\end{array}$ & $\begin{array}{l}\text { Male: } 10 \text {, Female: } 11 \\
\text { Age Range: } 25-83 \text { y.o. } \\
\text { (n/a separate data for } \\
\text { patients with } A B \\
\text { infections) }\end{array}$ & $\begin{array}{l}\text { BSI }(n=1), \text { SSTI } \\
(n=2), \text { LRTI }(n= \\
2)(N=5)\end{array}$ & $5(\mathrm{n} / \mathrm{a})$ & $\begin{array}{ll}- & \text { MDR-AB } \\
\text { - } & \text { Not defined } \\
& \text { E-test } \\
\end{array}$ & $\begin{array}{l}100 \mathrm{mg} \text { I.V. B.I.D., } \\
\text { converted to P.O. } \\
\text { per physician } \\
\text { decision }\end{array}$ & Not applicable & Not applicable & Not defined. & Not defined. \\
\hline $\begin{array}{l}\text { Study B, Chan } \\
\text { J.D.et al., } 2010 \text { [33] }\end{array}$ & $\begin{array}{l}\text { Male: 40, Female: } 15 \\
\text { Age Range: } 18-87 \text { y.o. }\end{array}$ & $\begin{array}{l}\mathrm{VAP} \\
(N=55)\end{array}$ & $36(11)$ & $\begin{array}{ll}\text { - } & \text { CR-AB } \\
\text { - } & \text { Not defined. } \\
& \text { Not reported. }\end{array}$ & $\begin{array}{l}200 \mathrm{mg} \text { LD } \\
\text { followed by } 100 \\
\text { mg I.V. B.I.D. OR } \\
200 \mathrm{mg} \text { P.O. B.I.D. }\end{array}$ & $\begin{array}{l}\operatorname{AMG}(n=20), \text { AMG + } \\
\text { TGC }(n=3), \text { AMG + } \\
\operatorname{PLM}(n=2)\end{array}$ & $\begin{array}{l}\text { TGC: } 100 \mathrm{mg} \text { LD - then } 50 \mathrm{mg} \\
\text { I.V. B.I.D., TOB: } 7 \mathrm{mg} / \mathrm{kg} \text { I.V. } \\
\text { OD. OR } 300 \mathrm{mg} \text { INH B.I.D., } \\
\text { GEN: } 7 \mathrm{mg} / \mathrm{kg} \text { I.V. OD, AMK: } \\
15 \mathrm{mg} / \mathrm{kg} \text { I.V. OD, PLM-B: } 2.5-5 \\
\mathrm{mg} / \mathrm{kg} \text { per day B.I.D. or Q.I.D., } \\
\text { COL: } 2.5-5 \mathrm{mg} / \mathrm{kg} \text { per day } \\
\text { B.I.D. or Q.I.D. or } 150 \mathrm{mg} \text { INH } \\
\text { B.I.D., SAM: } 2+1 \text { gr I.V. Q.I.D. }\end{array}$ & $\begin{array}{l}\text { Improvement and } \\
\text { resolution of signs and } \\
\text { symptoms of VAP. }\end{array}$ & $\begin{array}{l}\text { Eradication of CR-AB } \\
\text { from subsequent } B A L \\
\text { or sputum culture at } \\
\text { the completion of } \\
\text { therapy. }\end{array}$ \\
\hline $\begin{array}{l}\text { Study C, Goff D.A. } \\
\text { et al., } 2014 \text { [34] }\end{array}$ & $\begin{array}{l}\text { Male: 36, Female: } 19 \\
\text { Age Range: } 23-85 \text { y.o. }\end{array}$ & $\begin{array}{l}\text { LRTI }(n=32), \text { BSI } \\
(n=10), \text { IAI }(n= \\
\text { 3), LRTI BSI }(n= \\
\text { 4), SSTI }(n= \\
\text { 2), Osteomyelitis } \\
(n=2), \text { UTI }(n=2) \\
(N=55)\end{array}$ & $55(3)$ & $\begin{array}{ll}\text { - } & \text { MDR-AB } \\
\text { Non-susceptible to } \geq 1 \\
\text { antibiotic agent in } \\
\\
\geq 3 \text { categories. } \\
\text { - } & \text { E-test }\end{array}$ & $\begin{array}{l}100 \mathrm{mg} \text { I.V. B.I.D. } \\
(n=42 \text { received } \\
\text { 200mg I.V. LD) }\end{array}$ & $\begin{array}{l}\text { COL I.V. }(n=19) \\
\text { DOR + COL I.V. }(n= \\
\text { 9), SAM+COL I.V. }(\mathrm{n} \\
=7) \text {, DOR+COOL INH } \\
(n=4), \text { SAM }(n=3) \\
\text { SAM + DOR + COL } \\
\text { I.V. }(n=3) \text { DOR }(n= \\
\text { 2) SAM + DOR }(n=2) \\
\text { SAM + COL INH }(n \\
=1), \text { COL INH }(n=1) \\
\text { SAM + COL I.V.+ } \\
\text { COL INH }(n=1)\end{array}$ & Not applicable & $\begin{array}{l}\text { Complete or partial } \\
\text { resolution of the signs } \\
\text { \& symptoms } \\
\text { attributable to the } \\
\text { MDR-AB infection } \\
\text { without a need for the } \\
\text { escalation of } \\
\text { antimicrobials. }\end{array}$ & $\begin{array}{l}\text { Eradication of MDR-AB } \\
\text { in follow-up cultures } \\
\text { from the primary } \\
\text { source of infection } \\
\text { during treatment or } \\
\text { presumed when patient } \\
\text { clinically improved \& } \\
\text { follow-up cultures were } \\
\text { not performed. }\end{array}$ \\
\hline $\begin{array}{l}\text { Study } D \text {, Griffith } \\
\text { M.E. et al., } 2008 \\
\text { [35] }\end{array}$ & $\begin{array}{l}\text { Male: } 8 \\
\text { Age Range: 19-35 y.o. }\end{array}$ & $\begin{array}{l}\text { Osteomyelitis \& } \\
\text { SSII } \\
(N=8)\end{array}$ & $8(1)$ & $\begin{array}{ll}\text { - } & \text { MDR-ACB } \\
\text { Resistance to all } \\
\text { commonly tested } \\
\text { Cephalosporins, } \\
\beta \text {-Lactam } \\
\text { / } \beta \text {-Lactamase } \\
\text { Inhibitor } \\
\quad \& \text { Fluoroquinolones. } \\
\text { - } & \text { Disk Diffusion/BMD }\end{array}$ & 100 mg P.O. B.I.D. & $\begin{array}{l}\text { VAN }(n=2) \text {, AMK }(n \\
=1) \operatorname{IPM}(n=4)\end{array}$ & Not applicable & $\begin{array}{l}\text { No further clinical } \\
\text { evidence of infection as } \\
\text { determined by } \\
\text { symptoms, physical } \\
\text { examination, laboratory } \\
\text { evaluation. }\end{array}$ & Not defined \\
\hline $\begin{array}{l}\text { Study } E \text {, Ning F.et } \\
\text { al., } 2014 \text { [36] }\end{array}$ & $\begin{array}{l}\text { Male: 6, Female: } 3 \\
\text { Age Range: } 23-57 \text { y.o. }\end{array}$ & $\begin{array}{l}\text { LRTI }(n=3), \text { LRTI } \\
\text { + SSTI }(n=2), \\
\text { LRTI + SSTI + BSI } \\
(n=4) \\
(\boldsymbol{N}=\mathbf{9})\end{array}$ & $9(0)$ & $\begin{array}{ll}\text { - } & \text { PDR-AB } \\
\text { - Resistant to all } \\
\text { antibiotics except } \\
\text { PLM B. } \\
\text { - } & \text { Not reported }\end{array}$ & 200 mg P.O. Q.I.D. & $\begin{array}{l}\text { MEM I.V. Q.I.D. } \\
\text { (6gr/day } \\
\text { total).+CFP/S } 6 \text { gr I.V } \\
\text { B.I.D. }\end{array}$ & Not applicable & $\begin{array}{l}\text { Drugs were withdrawn } \\
\text { after symptoms' } \\
\text { improvement } \\
\text { combined with } \\
\text { negative blood \& } \\
\text { sputum cultures. }\end{array}$ & $\begin{array}{l}\text { Negative blood and } \\
\text { sputum cultures. }\end{array}$ \\
\hline
\end{tabular}


Table 3. Cont

\begin{tabular}{|c|c|c|c|c|c|c|c|c|c|}
\hline $\begin{array}{l}\text { Abbreviation ID } \\
\text { of study, Authors, } \\
\text { Year, Reference }\end{array}$ & $\begin{array}{l}\text { Patient } \\
\text { Demographics }\end{array}$ & $\begin{array}{l}\text { A. baumannii } \\
\text { Infections }(N= \\
\text { Total No. of } \\
\text { Patients) }\end{array}$ & $\begin{array}{l}\text { No. of } \\
\text { Patients on } \\
\text { Minocycline } \\
\text { (Monotherapy) }\end{array}$ & $\begin{array}{l}\text { Organisms, Resistance } \\
\text { Pattern Definition \& } \\
\text { Susceptibility Testing } \\
\text { Method }\end{array}$ & $\begin{array}{l}\text { Minocycline } \\
\text { Route of } \\
\text { Administration } \\
\text { \& Doses } \\
\end{array}$ & $\begin{array}{l}\text { Minocycline } \\
\text { Combinations }\end{array}$ & $\begin{array}{l}\text { Other Antimicrobial Agents } \\
\text { \& Doses }\end{array}$ & $\begin{array}{l}\text { Clinical Success } \\
\text { Definition }\end{array}$ & $\begin{array}{l}\text { Microbiological } \\
\text { Success Definition }\end{array}$ \\
\hline $\begin{array}{l}\text { StudyF, Pogue } \\
\text { J.M. et al., } 2014 \\
\text { [37] }\end{array}$ & $\begin{array}{l}\text { Male: } 5 \text {, Female: } 2 \\
\text { Age Range: } 35-74 \text { y.o. }\end{array}$ & $\begin{array}{l}\text { BSI }(n=3), \text { LRTI } \\
(n=3), \text { LRTI }+ \\
\text { SSTI }(n=1) \\
(N=7)\end{array}$ & $7(1)$ & $\begin{array}{ll}\text { - } & \text { CR-AB } \\
\text { - } & \text { Resistant to all CRB. } \\
& \text { E-test }\end{array}$ & $\begin{array}{l}100 \mathrm{mg} \text { I.V. B.I.D. } \\
(n=2) \\
200 \mathrm{mg} \text { I.V. B.I.D. } \\
(n=5)\end{array}$ & $\begin{array}{l}\mathrm{MEM}+\mathrm{COL}(n=1), \\
\mathrm{COL}(n=4), \operatorname{SAM}(n \\
=1)\end{array}$ & Not applicable & $\begin{array}{l}\text { Resolution of signs \& } \\
\text { symptoms of the } \\
\text { infection requiring } \\
\text { MIN. }\end{array}$ & $\begin{array}{l}\text { Clearance of the } \\
\text { organism of interest } \\
\text { from repeated cultures. }\end{array}$ \\
\hline $\begin{array}{l}\text { Study G, Shi Y. et } \\
\text { al., } 2012[38]\end{array}$ & $\begin{array}{l}\text { Male: } 49 \text {, Female: } 28 \\
\text { Age Range: } 49-89 \text { y.o. }\end{array}$ & $\begin{array}{l}\text { LRTI }(n=61), \text { BSI } \\
(n=5), \\
\text { Intra-abdominal } \\
(n=3), \text { SSTI }(n= \\
2), \text { Multiple sites } \\
(n=6) \\
(N=77)\end{array}$ & $77(0)$ & $\begin{array}{ll}\text { - } & \text { XDR-AB } \\
\text { - } & \text { Sensitive only to } 1 \text { or } \\
\text { - } & \text { classes of antibiotics. } \\
& \text { n/a }\end{array}$ & $\begin{array}{l}100 \mathrm{mg} \text { I.V. or P.O. } \\
\text { B.I.D. }\end{array}$ & $\begin{array}{l}\mathrm{CFP} / \mathrm{S}(n=70) \mathrm{CFP} / \mathrm{S} \\
+\operatorname{IPM} / \mathrm{CIL}(n=7)\end{array}$ & Not applicable & $\begin{array}{l}\text { Clinical effect divided } \\
\text { in: cure, marked } \\
\text { improvement, } \\
\text { improvement \& } \\
\text { ineffectiveness. } \\
\text { Effective group defined } \\
\text { as cure+marked } \\
\text { improvement. }\end{array}$ & Cleared cultures. \\
\hline $\begin{array}{l}\text { Study } H \text {, Wang } \\
\text { L.W. et al., } 2014 \\
\text { [39] }\end{array}$ & Mean age: $89.1+/-3.2$ & $\begin{array}{l}\text { VAP } \\
(N=42)\end{array}$ & $20(0)$ & $\begin{array}{ll}\text { - } & \text { PDR-AB } \\
\text { - } & \text { Not defined. } \\
\text { n/a }\end{array}$ & $\mathrm{n} / \mathrm{a}$ & $\mathrm{CFP} / \mathrm{S}$ & $\mathrm{n} / \mathrm{a}$ & Not defined & $\begin{array}{l}\text { Bacterial eradication of } \\
\text { PDR-AB from cultures. }\end{array}$ \\
\hline $\begin{array}{l}\text { Study I, Wood } \\
\text { G.C. et al., } 2003 \\
\text { [40] }\end{array}$ & $\begin{array}{l}\text { Male: } 7 \\
\text { (age n/a) }\end{array}$ & $\begin{array}{l}\operatorname{VAP}(n=5), \operatorname{VAP} \\
+\operatorname{BSI}(n=1), \operatorname{VAP} \\
+\operatorname{SSTI}(n=1) \\
(\boldsymbol{N}=7)\end{array}$ & $4(2)$ & $\begin{array}{ll}\text { - } & \text { MDR-AB } \\
\text { Resistance to all } \\
\text { antibiotics including } \\
\text { IPM-CIL and SAM. } \\
\text { - } \\
\text { VITEK }\end{array}$ & 100 mg I.V. B.I.D. & $\begin{array}{l}\text { IPM-CIL }(n=1) \text { TVA } \\
+ \text { TMP/SMX }(n=1) .\end{array}$ & DOX 100 mg I.V. B.I.D. & $\begin{array}{l}\text { AB was absent from the } \\
\text { follow-up BAL cultures } \\
\text { and the patient } \\
\text { improved clinically } O R \\
\text { The patient improved } \\
\text { clinically\& survived } \\
\text { until discharge. }\end{array}$ & $\begin{array}{l}\mathrm{AB} \text { was absent from the } \\
\text { follow-up } \mathrm{BAL} \\
\text { cultures. }\end{array}$ \\
\hline $\begin{array}{l}\text { Study J, Vila A. et } \\
\text { al., } 2016 \text { [41] }\end{array}$ & $\begin{array}{l}\text { Male: } 1 \text {, Female: } 2 \\
\text { Age Range: } 45-75 \text { y.o. }\end{array}$ & $\begin{array}{l}\text { PJI }(n=3) \\
(N=3)\end{array}$ & $2(0)$ & $\begin{array}{ll}\text { - } & \text { MDR-AB } \\
\text { - } & \text { Non- susceptible to } \\
& \geq 3 \text { categories. } \\
\text { - } & \text { Disk diffusion }\end{array}$ & 200 mg P.O/day & $\begin{array}{l}\text { TGC + COL (prior to } \\
\text { oral minocycline) } \\
(n=2)\end{array}$ & $\begin{array}{l}\text { TGC: } 100 \mathrm{mg} \text { LD - then } 100 \mathrm{mg} \\
\text { I.V. B.I.D, COL }\end{array}$ & $\begin{array}{l}\text { No signs and } \\
\text { symptoms of infection, } \\
\text { CRP }<10 \mathrm{mg} / \mathrm{L} \text {, normal } \\
\text { ESR, absence of } \\
\text { radiological signs of } \\
\text { loosening at the end of } \\
\text { treatment, without } \\
\text { further recurrences. }\end{array}$ & $\begin{array}{l}\text { Negative tissue cultures } \\
\text { from subsequent } \\
\text { debridements. }\end{array}$ \\
\hline
\end{tabular}


Documented A. baumannii infections treated either with a minocycline-based regimen or with other antimicrobial agents are shown in Table 3 . The vast majority of these infections were pneumonias $(\mathrm{n}=216,80.6 \%), 5$ with concomitant bacteremia and 4 with concomitant skin structure infections. $109(50.4 \%)$ patients were mechanically ventilated and treated for VAP. In addition, 19 (7.1\%) patients were treated for bloodstream infections, osteomyelitis and prosthetic joint infection were documented in $13(4.9 \%)$ patients, $6(2.2 \%)$ patients had wound/surgical site/SSTIs and another $6(2.2 \%)$ patients were diagnosed with complicated intra-abdominal infections (cIAIs). Multiple sites $(\geq 3)$ were documented in $10(3.7 \%)$ patients, 4 of whom had pneumonia, bacteremia and a wound infection simultaneously. Finally, one study reported two episodes of complicated urinary tract infection (study C).

In total, $223(83.2 \%)$ patients received minocycline as a monotherapy or in combination (Table 3), whereas the remaining $45(16.8 \%)$ were treated with other agents. In the minocycline group, monotherapy and combination was administered in 18 (8.3\%) and $200(91.7 \%)$ out of 218 patients with the available data, respectively. The dose of minocycline in most studies was $100 \mathrm{mg}$ twice daily (either in oral or in intravenous formulation) with or without a loading dose of $200 \mathrm{mg}$, with the exception of studies $E$ and $F$ where the doses were higher $(200 \mathrm{mg}$ four times and two times per day, respectively). The reason of the high dose administration was not sufficiently justified by the authors. The most frequent antimicrobials used in combination with minocycline were colistin (intravenous or inhaled), polymyxin B, cefoperazone/sulbactam and carbapenems (meropenem, doripenem and imipenem $+/-$ cilastatin). From the 200 patients on a minocycline - based combination regimen, $26(13 \%)$ received also colistin or polymyxin B; $14(7 \%)$ were on a colistin plus a carbapenem combination; cefoperazone/sulbactam was combined with minocycline in 90 patients (45\%) whereas in $7(3.5 \%)$ patients minocycline was combined with a carbapenem. Finally, $16(8 \%)$ were treated with a carbapenem plus cefoperazone/sulbactam plus minocycline. The remaining 47 (23.5\%) patients received a minocycline combination with other antimicrobials. The majority of the subjects were on a sulbactam-based combination $(n=124,62 \%)$. From the provided data, the duration of the minocycline administration varied significantly between the studies, with a range of 2 days to 3.3 months depending on the site of infection.

Non-minocycline based treatments were administered in 45 subjects. All patients were treated for A. baumannii associated VAP, except one patient who suffered from concurrent $A$. baumannii VAP and wound infection and one patient with a prosthetic joint infection. The most frequently administered antibiotics were aminoglycocides, either alone or in combination (13 out of 23 patients with the available data, $56.5 \%)$.

\subsection{Effect of Intervention on Outcomes}

Although the results in terms of outcomes were documented in all studies, the definitions of clinical and microbiological success varied across the studies (Table 3).

The outcomes were divided into two major groups: the group of patients who received a minocycline-based regimen either as monotherapy or in combination with other antimicrobials ("minocycline group") and the group where no minocycline was administered ("other antimicrobials group") and are summarized in Table 4. 
Table 4. Outcomes of included clinical studies.

\begin{tabular}{|c|c|c|c|c|c|c|c|c|}
\hline \multirow{2}{*}{$\begin{array}{l}\text { Abbreviation ID of } \\
\text { Study, Authors, } \\
\text { Year, Reference }\end{array}$} & \multicolumn{2}{|c|}{ Clinical Success } & \multicolumn{2}{|c|}{ Microbiological Success } & \multicolumn{2}{|c|}{ Mortality } & \multicolumn{2}{|c|}{ Adverse Events } \\
\hline & Minocycline (\%) & $\begin{array}{l}\text { Other Antibiotics } \\
(\%)\end{array}$ & $\begin{array}{l}\text { Minocycline } \\
\text { (\%) }\end{array}$ & $\begin{array}{l}\text { Other } \\
\text { Antibiotics (\%) }\end{array}$ & $\begin{array}{l}\text { Minocycline } \\
(\%)\end{array}$ & $\begin{array}{l}\text { Other } \\
\text { Antibiotics (\%) }\end{array}$ & $\begin{array}{l}\text { Minocycline } \\
\text { (\%) }\end{array}$ & $\begin{array}{l}\text { Other Antibiotics } \\
(\%)\end{array}$ \\
\hline $\begin{array}{l}\text { Study A, Bishburg E. } \\
\text { et al., 2014 [32] }\end{array}$ & $\begin{array}{l}5(100) \\
\text { BSI: } 1 \text { (100)LRTI: } 2 \\
\text { (100) }\end{array}$ & $\mathrm{n} / \mathrm{a}$ & Not available & $\mathrm{n} / \mathrm{a}$ & 0 & $\mathrm{n} / \mathrm{a}$ & Not available & $\mathrm{n} / \mathrm{a}$ \\
\hline $\begin{array}{l}\text { Study B, Chan J.D. } \\
\text { et al., 2010[33] }\end{array}$ & $\begin{array}{l}29(80.5) \\
V A P: 29(80.5)\end{array}$ & $13(68.4)$ & $\begin{array}{l}\text { Not separately } \\
\text { specified * }\end{array}$ & $\begin{array}{l}\text { Not separately } \\
\text { specified * }\end{array}$ & $\begin{array}{l}\text { Not separately } \\
\text { specified * }\end{array}$ & $\begin{array}{l}\text { Not separately } \\
\text { specified * }\end{array}$ & 0 & $\begin{array}{l}\text { Nephrotoxicity due to } \\
\text { aminoglycosides or } \\
\text { polymyxins: } 10 \text { (18.2) }\end{array}$ \\
\hline $\begin{array}{l}\text { Study C, Goff D.A. } \\
\text { et al., } 2014 \text { [34] }\end{array}$ & $\begin{array}{l}40(72.7) \\
\text { LRTI: } 20(62.5) \S \\
\text { BSI: } 9(90) \S \\
\text { LRTI+BSI: } 3(75) \S\end{array}$ & $\mathrm{n} / \mathrm{a}$ & $43(78)^{ \pm}$ & $\mathrm{n} / \mathrm{a}$ & $14(25.5)$ & $\mathrm{n} / \mathrm{a}$ & 0 & $\mathrm{n} / \mathrm{a}$ \\
\hline $\begin{array}{l}\text { Study D, Griffith } \\
\text { M.E. et al., } 2008 \text { [35] }\end{array}$ & $7(87.5)$ & $\mathrm{n} / \mathrm{a}$ & Not available & $\mathrm{n} / \mathrm{a}$ & 0 & $\mathrm{n} / \mathrm{a}$ & $\begin{array}{l}\text { Eosinophilia \& } \\
\text { neutropenia: } 1 \\
\text { (12.5) }\end{array}$ & $\mathrm{n} / \mathrm{a}$ \\
\hline $\begin{array}{l}\text { Study E, Ning F. et } \\
\text { al., } 2014 \text { [36] }\end{array}$ & $\begin{array}{l}9 \text { (100) } \\
\text { LRTI: } 3 \text { (100) LRTI + } \\
\text { SSTI: } 2 \text { (100) LRTI + } \\
\text { SSTI + BSI: } 4 \text { (100) }\end{array}$ & $\mathrm{n} / \mathrm{a}$ & $8(88.9)^{ \pm}$ & $\mathrm{n} / \mathrm{a}$ & 0 & $\mathrm{n} / \mathrm{a}$ & 0 & $\mathrm{n} / \mathrm{a}$ \\
\hline $\begin{array}{l}\text { Study F, Pogue J.M. } \\
\text { et al., } 2014 \text { [37] }\end{array}$ & $\begin{array}{l}5 \text { (71.4) } \\
\text { LRTI: } 1(50) \\
\text { BSI: } 2 \text { (66.6) } \\
\text { LRTI + SSTI: } 1 \text { (100) } \\
\text { LRTI + BSI: } 1 \text { (100) }\end{array}$ & $\mathrm{n} / \mathrm{a}$ & $\begin{array}{l}3(60) * * \\
\text { LRTI + BSI: } 1 \\
(100) \\
\text { BSI: } 2(66.6)\end{array}$ & $\mathrm{n} / \mathrm{a}$ & $2(28.6)$ & $\mathrm{n} / \mathrm{a}$ & $\begin{array}{l}\text { Nephrotoxicity } \\
\text { due to colistin } \\
\text { combination: } \\
2(28.6)\end{array}$ & $\mathrm{n} / \mathrm{a}$ \\
\hline $\begin{array}{l}\text { Study G, Shi Y. et al., } \\
2012 \text { [38] }\end{array}$ & $48(62.3)^{ \pm}$ & $\mathrm{n} / \mathrm{a}$ & $36(46.8)^{ \pm}$ & $\mathrm{n} / \mathrm{a}$ & $19(24.7)$ & $\mathrm{n} / \mathrm{a}$ & $\begin{array}{l}\text { Elevation of } \\
\text { LFTs: } \\
6(7.8)\end{array}$ & $\mathrm{n} / \mathrm{a}$ \\
\hline $\begin{array}{l}\text { Study H, Wang L.W. } \\
\text { et al., } 2014 \text { [39] }\end{array}$ & $\begin{array}{l}13(65) \\
\text { VAP: } 13 \text { (65) }\end{array}$ & $\begin{array}{l}5(22.7) \\
\operatorname{VAP}: 5(22.7)\end{array}$ & $\begin{array}{l}8(40) \\
\text { VAP: } 8(40)\end{array}$ & $\begin{array}{l}3(13.6) \\
\operatorname{VAP}: 3(13.6)\end{array}$ & Not available & Not available & Not available & Not available \\
\hline $\begin{array}{l}\text { Study I, Wood G.C. } \\
\text { et al., } 2003 \text { [40] }\end{array}$ & $\begin{array}{l}4(100) \\
\operatorname{VAP}: 4(100)^{ \pm}\end{array}$ & $\begin{array}{l}2(66.7) \\
\operatorname{VAP:} 2(66.7)^{ \pm}\end{array}$ & $\begin{array}{l}3(100)^{* *} \\
V A P: 3(100)^{ \pm}\end{array}$ & $\begin{array}{l}1(50)^{* *} \\
\operatorname{VAP:} 1(50)^{ \pm}\end{array}$ & 0 & $1(33.3)$ & 0 & 0 \\
\hline $\begin{array}{l}\text { Study J, Vila A. et } \\
\text { al., 2016 [41] }\end{array}$ & $2(100)$ & $1(100)$ & $2(100)$ & $1(100)$ & 0 & 0 & 0 & 0 \\
\hline
\end{tabular}


In the minocycline group $(n=223)$, the overall reported clinical success rate was $72.6 \%(n=162)$ for all documented infections. The clinical success rates ranged between $62.3 \%$ and $100 \%$ among the individual studies; the highest rates were reported in three small USA studies and one study from Argentina, whereas the lower ones were documented in two Chinese studies. A clinical cure was documented in 16 out of $18(88.9 \%)$ and in 141 out of $200(70.5 \%)$ patients (with available data) who received minocycline monotherapy and combination therapy respectively. Microbiological data were available for $171(76.7 \%)$ patients in the minocycline group $(n=223)$; among them, $103(60.2 \%)$ had a documented or presumed microbiological success.

Lower respiratory tract infection was the most prevalent type of infection. In total, 172 out of $216(79.6 \%)$ pneumonias (with or without another concomitant $A$. baumannii infection) were treated with a minocycline-based regimen. Clinical success was reported in $121(70.3 \%)$ patients. For the 65 minocycline- treated VAPs in particular, the success rate was $78.5 \%(n=51)$. Additionally, from 28 documented bloodstream infections (alone or combined with other sites of infection) in the minocycline group, a clinical cure was obtained in 22 patients (78.6\%). Clinical success was reported in 22 out of 25patients ( $88 \%$ ) with osteomyelitis, prosthetic joint infections and/or SSTIs (alone or combined with other sites of infection) who received minocycline either as a monotherapy or in combination with other antimicrobials. Mortality rates were available in 8 studies. From 167 patients with relevant data, 35 patients died resulting in a mortality rate of $20.9 \%$. All deaths were clinically judged as being associated with the primary infection and, therefore, they were considered as clinical failures of the minocycline. Finally, the data for hospital readmissions were not available in any of the evaluated studies.

In the non-minocycline group, a clinical cure was obtained in 21 (46.7\%) patients. All infections in this group were VAP, except in study $J$ that included one prosthetic joint infection. A microbiological cure was reported in 5 out of $26(19.2 \%)$ with available data.

Adverse events in the minocycline group were reported in 9 patients (4\%). Namely, 2 patients experienced an acute kidney injury likely attributable to the co-administered colistin. One patient developed neutropenia and eosinophilia whilst on the minocycline treatment. Importantly, an increase of liver function tests (LFTs) and abnormally high coagulation times was observed in 6 subjects from study $G$ that included patients with cIAIs.

\section{Discussion}

This review aimed to systematically evaluate the existing evidence on minocycline effectiveness in the treatment of nosocomial infections caused by MDR, XDR and PDR A. baumannii strains. In brief, among 268 adult patients with a variety of nosocomial infections caused by MDR, XDR or PDR A. baumannii strains, 223 patients received a minocycline-based regimen with an overall documented clinical success rate of $72.6 \%$. The majority of the patients received a minocycline combination, with a clinical success rate of $70.5 \%$, compared to $88.9 \%$ in the monotherapy counterpart. The most prevalent infection in this study population was lower respiratory tract infection, including VAP $(80.6 \%)$. The observed clinical cure rate among pneumonias was $70.3 \%$, whereas for VAP in particular, the reported success rate was $78.5 \%$. The microbiological success rate was $60.2 \%$, and the crude mortality rate in the minocycline group was $20.9 \%$. The results of the current systematic review suggest that minocycline may be an effective antimicrobial agent in the treatment of resistant $A$. baumannii nosocomial infections. However, it would be risky to draw robust conclusions through direct comparisons as no relevant adjustments have been made for the two evaluated groups of treatment. In this review, $91.7 \%$ of the participants received a minocycline combination with one or more classes of other antimicrobial agents. It is quite interesting that minocycline monotherapy demonstrated higher percentages of clinical success compared to combination regimens. Whether the administration of combinations occurred in more severe underlying infections or sicker patients and therefore resulted in lower success rates, or indeed, whether minocycline is more effective as a monotherapy, cannot be answered from this data. Many microbiological studies have reported the synergistic effects of 
minocycline when combined with other agents like rifampicin, imipenem, cefoperazone/sulbactam or colistin in in vitro experiments [42-45]. Similarly, in animal studies, combination regimens with rifampicin, amikacin or polymyxin $\mathrm{B}$ have also demonstrated promising results against experimental A. baumannii infections [46,47].

As opposed to the in vitro and animal data, the evidence for the advantages of combination therapies over monotherapies in the treatment of MDROs in clinical practice is not vigorous and is still being explored [48]. A meta-analysis of colistin monotherapy versus colistin combinations in the treatment of carbapenem resistant gram-negative bacteria reported that the subgroups of colistin/carbapenem, colistin/rifampicin and colistin/tigecycline did not demonstrate any benefits in mortality over monotherapy [49]. Correspondingly, a systematic review of combination therapies versus monotherapies for MDR, XDR and PDR A. baumannii infections was rather inconclusive in terms of superiority between the two therapeutic approaches [50]. However, the use of combinations does not come without consequences. The utilization of possibly non-effective antimicrobials in therapeutic schemes, based on in vitro synergistic effects, may induce or maintain colonization with more resistant bacteria, contributes to Clostridium difficile and fungal infections and increases the antibiotic-related adverse events $[49,51]$.

On examining the clinical outcomes of this review, a diversity of clinical success rates is observed between two Chinese and the remaining studies, with the former ones reporting the lowest success rates. Whether this could be attributable to the advanced resistance profile of their isolates or just a confounder of the patient populations' baseline characteristics, is not clear. Nonetheless, the regional use of minocycline in each country and its consequential impact on the susceptibility rates should be taken into account. The global data suggest that resistance has been continuously rising, even for last-resort antibiotics like polymyxins; thus, the total stock of antibiotic effectiveness steadily declines [52]. Sensibly, the pattern of microbial resistance between and within the countries is reflecting the patterns of the antibiotic use and the regional infectious diseases [52]. Therefore, it may be reasonable to say that minocycline susceptibility patterns would follow the same norm, whereby countries with a higher consumption would exhibit higher resistance rates, but more evidence to support this is necessary. The overall success rates of minocycline are in line with the existing literature. Similar success rates are reported in studies of VAP treated with combinations of $\beta$-lactams, or colistin or tigecycline, when the pathogen is susceptible to the administered regimen [53-55]. The mortality rate of the minocycline treated group was $20.9 \%$. All deaths were associated with the primary infection and, therefore, they were considered as clinical failures of the minocycline-based regimen. Importantly, it is not reported by the authors whether these failures were attributable to the development of a minocycline resistance during the treatment or to the severity of the underlying infection. These data are highly important. There is a large debate on the mortality associated with A. baumannii infections; hence, the results of this review are again within the expected ranges [56,57]. Like the threat of plasmid mediated colistin resistance through the MCR-1 gene and the ongoing debate about increased mortality with tigecycline in critically ill ICU patients, the results of minocycline use reported here, are a welcome addition to our limited armamentarium and a possible trigger for further research [56,58-63].

The microbiological success rates in this study population were considerably lower than the relevant clinical rates. This may reflect the practical difficulties in pursuing follow-up samples in order to determine the bacterial eradication. Nevertheless, the role of A. baumannii as a colonizer cannot be overlooked. Many studies have demonstrated high prevalence rates in the colonization by resistant A. baumannii strains among immunocompromised patients with severe co-morbidities, like critically ill patients or residents of nursing homes [64-67]. These studies highlighted the epidemiologic profile of patients at risk of $A$. baumannii acquisition. Nevertheless, clinicians often face the dilemma of deciding when a bystander becomes a pathogen. Although in most cases the decision to treat is made upon individualized criteria, there is inadequate evidence to determine the transition of an isolate from a colonizer into a pathogen. The persistence of the pathogen in clinically non-significant counts has been reported in studies of VAP caused by P. aeruginosa and A. baumannii. This phenomenon may 
be attributed to the presence of biofilm-embedded bacterial populations, which are quite difficult to eradicate with currently achievable concentrations of antibiotics in deep lung tissues by the conventional intravenous route [68].

The growing issue of multidrug resistant Acinetobacter strains is firmly acknowledged, and the multifaceted consequences are now well-known to the medical community. This phenomenon is partially owed to the lack of large RCTs from the available literature. Minocycline in particular, although promising, has only been studied in observational studies. Therefore, the conduction of randomized trials, including minocycline in the evaluated regimens, along with other currently available agents, is imperative in order to create a solid base of evidence from which guidelines can be drawn. In an effort to address this difficult issue, a task force on the management of $A$. baumannii infections consisting of a multinational panel of experts, has recently issued a position paper on the management of $A$. baumannii infections in critically ill patients and particularly in the ICU setting [69]. Interestingly, monotherapy was recommended as a definite treatment of $A$. baumannii infections, irrespective of the source and type of the selected antibiotic, based on the lack of solid evidence supporting combination treatments as contributors to improved clinical outcomes [69]. According to the authors, "the combination of sulbactam or a polymyxin with a second agent (tigecycline, rifampicin, or fosfomycin) may be considered for clinical failures or for infections caused by an isolate with MIC in the upper limit of susceptibility" [69]. Minocycline is not recommended as a treatment option in this position paper [69].

Even with advanced antimicrobial regimens, MDR, XDR and PDR A. baumannii infections still carry high mortality rates and a considerable healthcare and economic burden. It seems that any treatment may be futile if the healthcare providers do not put efforts in impeding the spread of the A. baumannii strains. The more the clinicians administer extended-spectrum antibiotics to eliminate the increasingly resistant bacteria, the more "powerful" and resistant to available treatments those strains become. As currently there are no formulated, evidence-based guidelines that would direct not only the most active regimens, but also their doses and the duration of the treatment according to the site of the infection and the regional susceptibilities, the administered antibiotics may be suboptimal, thus inducing a vicious cycle of maintaining and enhancing the strains' resistance. Consequently, instead of solely trying to treat them, the role of reducing the dissemination of these isolates within the healthcare environment should not be disregarded. To be pre-emptive is a rule of thumb in medicine, and in this case, the infection control measures are the way to achieve this. The European Society of Clinical Microbiology and Infectious Diseases (ESCMID) and the CDC have defined infection control measures for multidrug resistant bacteria, including MDR/XDR Acinetobacter [70,71]. These measures have shown very encouraging results in controlling and reducing the observed outbreaks in healthcare settings and, therefore, they should be established as common practice in nosocomial environments [72-74].

Multiple methodological challenges were faced in the present study and the results should be interpreted in the light of certain limitations. All included studies in this review were observational case series reporting minocycline use in small patient populations. This affects not only the statistical strength of the available data, but also the analysis and the interpretation of the results that were analyzed through an integrative manner that lacks the statistical strength of a meta-analysis. Unfortunately, there is a paucity of randomized controlled trials (RCTs) and the generalization of the results should therefore be done cautiously. Furthermore, the analyzed clinical studies demonstrate a notable diversity of selection criteria and patient characteristics. Unequivocally, this generates a sampling selection bias not only at the study level, but also at the level of cumulating evidence. Indeed, the retrospective researcher-directed recruitment of subjects on a minocycline-oriented therapy, without a comparator group in most cases, enhances the chance of a selection effect that may interfere with the observed clinical outcomes. Additionally, most studies performed in the USA reported the use of the intravenous (IV) formulation of minocycline (that was re-introduced there in 2009) [32]. However, the IV formulation is unavailable in many countries worldwide. Only recently, intravenous minocycline was introduced for licensing in the Latin America countries [75]. This may affect the applicability of the results in 
clinical practice, as there are no available studies comparing the oral versus the IV formulation in critically ill patients with gram-negative MDROs. Furthermore, the vast majority of the patients in this analysis received a minocycline combination rather than minocycline monotherapy. This raises the likelihood that the reported results and outcomes in the minocycline group may be influenced by the co-administered agent, thus resulting in confounding bias. Although the evidence is scarce for minocycline combinations, in clinical practice combination regimens are usually preferred over monotherapies in the treatment of resistant A. baumannii infections [50,76-80]. Another possible limitation of this review is the observed inconsistency between the definitions used for the isolates' resistance among the selected trials. Indeed, only two studies (Goff et al. and Shi et al.) have defined the isolates in absolute concordance with the criteria set by established authorities dealing with antimicrobial resistance [30,34,38]. Even if not fully consistent with the synchronous terminology, these studies still fulfill the inclusion criteria as they involve strains that are at least MDR. All studies, except the one by Ning et al., evaluated strains that were either susceptible or with an intermediate susceptibility to minocycline [36]. However, only three included MIC data (Goff et al., Bishburg et al. and Pogue et al.) $[32,34,37]$. The susceptibility breakpoints were established using outdated methods that do not incorporate pharmacodynamics. There is growing evidence that broth microdilution (BMD) and VITEK 2 appear to be more reliable than an E-test for minocycline susceptibility testing in MDR Acinetobacter spp. [81].Variable susceptibility rates of MDR Acinetobacter spp., depending on the testing method, have been reported and MIC breakpoints may be lower than the ones currently used $[82,83]$. Furthermore, all included studies fail to report screening for the emergence of resistance to minocycline with repeat susceptibility testing. Interestingly, in the study by Chan et al., an intermediate resistance was reported in 4 out of 6 patients receiving tigecycline [33]. Further efforts in examining the minocycline use should address the potential for the emergence of resistance or cross-resistance with other drugs of the same class.

The mortality rate of MDR A. baumannii infections in this systematic review is considerably lower than previously reported data [14-19]. This bias is potentially introduced by the inclusion of less severe infections such as osteomyelitis and SSTIs. Additionally, the type of infection and the bioavailability may affect the activity of a static ribosomal targeting antibiotic like minocycline (BSI versus SSTI). Another major limitation was that the time of the appropriate antimicrobial treatment initiation (including minocycline) was not addressed in the individual studies. Nevertheless, one could say that earlier antimicrobial therapy in some studies may have introduced a selection bias, thus favoring the use of minocycline. Finally, the included studies reported only scarce data on the safety profile of the drug.

Previously published reviews examining the role of minocycline in the treatment of resistant A. baumannii strains demonstrated similar results, under the constriction of the same limitations $[7,76,84]$. Nevertheless, the current systematic review includes more clinical studies and thus a larger patient population. Furthermore, it examines the role of both intravenous and oral formulations of minocycline in the treatment of resistant $A$. baumannii strains. Eligible studies were carefully examined in order to avoid overlapped populations. One study that has been included in previous reviews was therefore excluded from the analysis [34,85].

Taking into account its limitations, this review demonstrates evidence that minocycline may be an alternative therapy for nosocomial infections caused by resistant $A$. baumannii strains. Treatment options for MDR, XDR and PDR A. baumannii strains are considerably limited and inversely proportional to the resistance profile. Few antibiotics remain active and effective enough to endeavor a successful outcome in nosocomial infections caused by these strains. Among them, sulbactam, polymyxins, tigecycline and a combination of different classes of antibiotics are frequently used, with polymyxins being the current mainstay of treatment [24]. The medical community is in need of more therapeutic approaches, beyond colistin, as the exclusive administration and overuse of one antimicrobial class will inevitably limit the susceptible bacterial population, thus nullifying one more last-resort agent. Therefore, the evidence of using alternative therapeutic agents (alone or in combination) for A. baumannii infections like 
tigecycline, colistin, and sulbactam-based regimens should not be overlooked [86-89]. Minocycline is unique among the currently available therapeutic options against MDR A. baumannii in that it can be administered orally. This can be particularly important for clinically stable patients requiring a prolonged treatment.. Newer tetracycline derivatives may have an additional role in the future therapeutics of MDR Acinetobacter [90].

\section{Conclusions}

The prompt and optimal use of antibiotics is the solution of the perpetual puzzle of multi-resistant bacteria like Acinetobacter. However, what constitutes the optimal treatment still remains unresolved, while at the same time, the emergence of more resistant and virulent strains compels the need for an answer. Minocycline could constitute an alternative agent for the treatment of MDR A. baumannii infections. The therapeutic niche for minocycline could involve switching to oral therapy for clinically stable patients that require a prolonged treatment. However, the available clinical data that we reviewed, although promising, are still inconclusive. Research efforts with randomized studies, that utilize standard definitions for MDR/XDR/PDR organisms, employ uniform dosing regimens, evaluate the time of onset of an appropriate antimicrobial therapy and assess for the emergence of resistance by repeat susceptibility testing, should be made to define the role of currently available antibiotics, like minocycline, in the treatment of $A$. baumannii infections as novel agents are still under development or evaluation.

Supplementary Materials: The following is available online at http://www.mdpi.com/2076-2607/7/6/159/s1: Table S1: The PRISMA-P2015 Checklist.

Author Contributions: Conceptualization, P.C.F., G.P. and S.T.; methodology, P.C.F. and D.K.M.; software, A.B., M.B., V.R., and D.E.K.; validation, P.C.F., G.P., A.P., D.K.M. and S.T.; formal analysis, P.C.F. and D.K.M.; investigation, P.C.F., G.P., A.P., A.B., M.B., V.R., D.K. and D.E.K.; resources, A.B., M.B., V.R., D.K. and D.E.K.; data curation, P.C.F., G.P. and S.T.; writing-original draft preparation, P.C.F., G.P., A.B., M.B.,V.R., D.K. and D.E.K.; writing-review and editing, S.T., D.K.M., D.E.K., D.K. and A.P., visualization, P.C.F.; supervision, S.T.; project administration, P.C.F.

Funding: This research received no external funding.

Conflicts of Interest: The authors declare no conflict of interest.

\section{Appendix A}

Table A1. The STROBE statement checklist scores for included studies.

\begin{tabular}{lll}
\hline $\begin{array}{l}\text { Abbreviation ID of study, Authors, } \\
\text { Year, Reference }\end{array}$ & Satisfied Criteria of the STROBE Checklist & Score \\
\hline Study A, Bishburg E. et al., 2014 [32] & $1(\mathrm{a}, \mathrm{b}), 2,3,4,5,6(\mathrm{a}), 7,8,10,11,13(\mathrm{a}), 14(\mathrm{a}), 15,18,19,20,21,22$ & $18 / 22$ \\
\hline Study B, Chan J.D. et al., 2010 [33] & $1(\mathrm{a}, \mathrm{b}), 2,3,4,5,6(\mathrm{a}), 7,8,10,11,13(\mathrm{a}), 14(\mathrm{a}), 15,17,18,19,20,21,22$ & $19 / 22$ \\
\hline Study C, Goff D.A. et al., 2014 [34] & $1(\mathrm{a}, \mathrm{b}), 2,3,4,5,6(\mathrm{a}), 7,8,10,11,13(\mathrm{a}), 14(\mathrm{a}), 15,17,18,19,20,21,22$ & $19 / 22$ \\
\hline Study D, Griffith M.E. et al., 2008 [35] & $1(\mathrm{a}, \mathrm{b}), 2,3,4,5,6 \mathrm{a}, 7,8,10,11,13 \mathrm{a}, 14(\mathrm{a}, \mathrm{b}), 15,17,18,19,20,21,22$ & $19 / 22$ \\
\hline Study E, Ning F.et al., 2014 [36] & $1(\mathrm{a}, \mathrm{b}), 2,3,4,5,6(\mathrm{a}), 7,8,10,11,12(\mathrm{a}, \mathrm{b}), 13(\mathrm{a}), 14(\mathrm{a}, \mathrm{b}), 15,17,18,19,20,21,22$ & $20 / 22$ \\
\hline Study F, Pogue J.M. et al., 2014 [37] & $1(\mathrm{a}, \mathrm{b}), 2,3,4,5,6(\mathrm{a}), 7,8,10,11,13(\mathrm{a}, \mathrm{b}), 14(\mathrm{a}, \mathrm{b}), 15,16(\mathrm{a}), 17,18,19,20,21,22$ & $20 / 22$ \\
\hline Study G, Shi Y. et al., 2012 [38] & $1(\mathrm{a}, \mathrm{b}), 2,3,4,5,6,7,8,9,10,11,12(\mathrm{a}, \mathrm{b}), 13(\mathrm{a}), 14(\mathrm{a}, \mathrm{b}), 15,16(\mathrm{a}), 17,18,19,20,21,22$ & $22 / 22$ \\
\hline Study H, Wang L.W. et al., 2014 [39] & $1(\mathrm{a}, \mathrm{b}), 2,3,4,5,6(\mathrm{a}), 7,8,10,11,13(\mathrm{a}), 14(\mathrm{a}), 15,18,19,20,21,22$ & $18 / 22$ \\
\hline Study I, Wood G.C. et al., 2003 [40] & $1(\mathrm{a}, \mathrm{b}), 2,3,4,5,6,7,8,10,13(\mathrm{a}, \mathrm{b}), 14(\mathrm{a}, \mathrm{b}), 15,17,18,19.20,21,22$ & $18 / 22$ \\
\hline StudyJ, Vila A. et al., 2016 [41] & $1(\mathrm{a}, \mathrm{b}), 2,3,4,5,6 \mathrm{a}, 7,8,10,11,13 a, 14(\mathrm{a}, \mathrm{b}), 15,17,18,19,20,21,22$ & $19 / 22$ \\
\hline
\end{tabular}




\section{References}

1. Redin, G.S. Antibacterial Activity in Mice of Minocycline, a New Tetracycline. Antimicrob. Agents Chemother. 1966, 6, 371-376. [PubMed]

2. Garrido-Mesa, N.; Zarzuelo, A.; Gálvez, J. Minocycline: Far beyond an Antibiotic. Br. J. Pharmacol. 2013, 169, 337-352. [CrossRef] [PubMed]

3. Chopra, I.; Roberts, M. Tetracycline Antibiotics: Mode of Action, Applications, Molecular Biology, and Epidemiology of Bacterial Resistance. Microbiol. Mol. Biol. Rev. 2001, 65, 232-260. [CrossRef] [PubMed]

4. Klein, N.C.; Cunha, B.A. Tetracyclines. Med. Clin. North Am. 1995, 79, 789-801. [CrossRef]

5. Agwuh, K.N.; MacGowan, A. Pharmacokinetics and Pharmacodynamics of the Tetracyclines Including Glycylcyclines. J. Antimicrob. Chemother. 2006, 58, 256-265. [CrossRef] [PubMed]

6. Smith, K.; Leyden, J.J. Safety of Doxycycline and Minocycline: A Systematic Review. Clin. Ther. 2005, 27, 1329-1342. [CrossRef]

7. Ritchie, D.J.; Garavaglia-Wilson, A. A Review of Intravenous Minocycline for Treatment of Multidrug-Resistant Acinetobacter Infections. Clin. Infect. Dis. 2014, 59, S374-S380. [CrossRef]

8. Santajit, S.; Indrawattana, N. Mechanisms of Antimicrobial Resistance in ESKAPE Pathogens. Biomed Res. Int. 2016, 2016, 1-8. [CrossRef]

9. Boucher, H.W.; Talbot, G.H.; Benjamin, D.K.; Bradley, J.; Guidos, R.J.; Jones, R.N.; Murray, B.E.; Bonomo, R.A.; Gilbert, D. Infectious Diseases Society of America. 10 x '20 Progress-Development of New Drugs Active Against Gram-Negative Bacilli: An Update from the Infectious Diseases Society of America. Clin. Infect. Dis. 2013, 56, 1685-1694. [CrossRef]

10. Antunes, L.C.S.; Visca, P.; Towner, K.J. Acinetobacter Baumannii: Evolution of a Global Pathogen. Pathog. Dis. 2014, 71, 292-301. [CrossRef]

11. Gerner-Smidt, P. Ribotyping of the Acinetobacter Calcoaceticus-Acinetobacter Baumannii Complex. J. Clin. Microbiol. 1992, 30, 2680-2685. [PubMed]

12. Bassetti, M.; Righi, E.; Esposito, S.; Petrosillo, N.; Nicolini, L. Drug Treatment for Multidrug-Resistant Acinetobacter Baumannii Infections. Future Microbiol. 2008, 3, 649-660. [CrossRef] [PubMed]

13. Manchanda, V.; Sinha, S.; Singh, N. Multidrug Resistant Acinetobacter. J. Glob. Infect. Dis. $2010,2,291$. [CrossRef] [PubMed]

14. Falagas, M.E.; Kopterides, P.; Siempos, I.I. Attributable Mortality of Acinetobacter Baumannii Infection among Critically Ill Patients. Clin. Infect. Dis. 2006, 43, 389-390. [CrossRef] [PubMed]

15. Lee, N.-Y.; Lee, H.-C.; Ko, N.-Y.; Chang, C.-M.; Shih, H.-I.; Wu, C.-J.; Ko, W.-C. Clinical and Economic Impact of Multidrug Resistance in Nosocomial Acinetobacter Baumannii Bacteremia. Infect. Control Hosp. Epidemiol. 2007, 28, 713-719. [CrossRef]

16. Abbo, A.; Carmeli, Y.; Navon-Venezia, S.; Siegman-Igra, Y.; Schwaber, M.J. Impact of Multi-Drug-Resistant Acinetobacter Baumannii on Clinical Outcomes. Eur. J. Clin. Microbiol. Infect. Dis. 2007, 26, 793-800. [CrossRef]

17. Falagas, M.; Bliziotis, I.; Siempos, I. Attributable Mortality of Acinetobacter Baumannii Infections in Critically Ill Patients: A Systematic Review of Matched Cohort and Case-Control Studies. Crit. Care 2006, 10, R48. [CrossRef]

18. Kwon, K.T.; Oh, W.S.; Song, J.-H.; Chang, H.-H.; Jung, S.-I.; Kim, S.-W.; Ryu, S.Y.; Heo, S.T.; Jung, D.S.; Rhee, J.-Y.; et al. Impact of Imipenem Resistance on Mortality in Patients with Acinetobacter Bacteraemia. J. Antimicrob. Chemother. 2007, 59, 525-530. [CrossRef]

19. Falagas, M.E.; Rafailidis, P.I. Attributable Mortality of Acinetobacter Baumannii: No Longer a Controversial Issue. Crit. Care 2007, 11, 134. [CrossRef]

20. Maragakis, L.L.; Perl, T.M. Antimicrobial Resistance: Acinetobacter Baumannii: Epidemiology, Antimicrobial Resistance, and Treatment Options. Clin. Infect. Dis. 2008, 46, 1254-1263. [CrossRef]

21. Bonomo, R.A.; Szabo, D. Mechanisms of Multidrug Resistance in Acinetobacter Species and Pseudomonas Aeruginosa. Clin. Infect. Dis. 2006, 43, S49-S56. [CrossRef] [PubMed]

22. Poirel, L.; Nordmann, P. Carbapenem Resistance in Acinetobacter Baumannii: Mechanisms and Epidemiology. Clin. Microbiol. Infect. 2006, 12, 826-836. [CrossRef] [PubMed] 
23. Pfeifer, Y.; Wilharm, G.; Zander, E.; Wichelhaus, T.A.; Göttig, S.; Hunfeld, K.-P.; Seifert, H.; Witte, W.; Higgins, P.G. Molecular Characterization of BlaNDM-1 in an Acinetobacter Baumannii Strain Isolated in Germany in 2007. J. Antimicrob. Chemother. 2011, 66, 1998-2001. [CrossRef] [PubMed]

24. Doi, Y.; Murray, G.; Peleg, A. Acinetobacter Baumannii: Evolution of Antimicrobial Resistance-Treatment Options. Semin. Respir. Crit. Care Med. 2015, 36, 085-098. [CrossRef]

25. Cai, Y.; Chai, D.; Wang, R.; Liang, B.; Bai, N. Colistin Resistance of Acinetobacter Baumannii: Clinical Reports, Mechanisms and Antimicrobial Strategies. J. Antimicrob. Chemother. 2012, 67, 1607-1615. [CrossRef]

26. Quale, J.; Shah, N.; Kelly, P.; Babu, E.; Backer, M.; Rosas-Garcia, G.; Salamera, J.; George, A.; Bratu, S.; Landman, D. Activity of Polymyxin B and the Novel Polymyxin Analogue CB-182,804 Against Contemporary Gram-Negative Pathogens in New York City. Microb. Drug Resist. 2012, 18, 132-136. [CrossRef]

27. Moher, D.; Shamseer, L.; Clarke, M.; Ghersi, D.; Liberati, A.; Petticrew, M.; Shekelle, P.; Stewart, L.A. Preferred Reporting Items for Systematic Review and Meta-Analysis Protocols (PRISMA-P) 2015 Statement. Syst. Rev. 2015, 4, 1. [CrossRef]

28. PRISMA. Available online: http://www.prisma-statement.org/PRISMAStatement/ (accessed on 1 February 2019).

29. Identifying Healthcare-Associated Infections (HAI) for NHSN Surveillance. Available online: https://www. cdc.gov/nhsn/PDFs/pscManual/2PSC_IdentifyingHAIs_NHSNcurrent.pdf (accessed on 2 February 2019).

30. Magiorakos, A.-P.; Srinivasan, A.; Carey, R.B.; Carmeli, Y.; Falagas, M.E.; Giske, C.G.; Harbarth, S.; Hindler, J.F.; Kahlmeter, G.; Olsson-Liljequist, B.; et al. Multidrug-Resistant, Extensively Drug-Resistant and Pandrug-Resistant Bacteria: An International Expert Proposal for Interim Standard Definitions for Acquired Resistance. Clin. Microbiol. Infect. 2012, 18, 268-281. [CrossRef]

31. Vandenbroucke, J.P.; von Elm, E.; Altman, D.G.; Gøtzsche, P.C.; Mulrow, C.D.; Pocock, S.J.; Poole, C.; Schlesselman, J.J.; Egger, M. STROBE Initiative. Strengthening the Reporting of Observational Studies in Epidemiology (STROBE): Explanation and Elaboration. PLoS Med. 2007, 4, e297. [CrossRef]

32. Bishburg, E.; Shah, M.; Chan, T. Use of Intravenous Minocycline for the Treatment of Methicillin-Resistant Staphylococcus Aureus (MRSA) and Resistant Gram-Negative Organisms. Infect. Dis. Clin. Pract. 2014, 22, 26-31. [CrossRef]

33. Chan, J.D.; Graves, J.A.; Dellit, T.H. Antimicrobial Treatment and Clinical Outcomes of Carbapenem-Resistant Acinetobacter Baumannii Ventilator-Associated Pneumonia. J. Intensive Care Med. 2010, 25, 343-348. [CrossRef] [PubMed]

34. Goff, D.A.; Bauer, K.A.; Mangino, J.E. Bad Bugs Need Old Drugs: A Stewardship Program's Evaluation of Minocycline for Multidrug-Resistant Acinetobacter Baumannii Infections. Clin. Infect. Dis. 2014, 59, S381-S387. [CrossRef] [PubMed]

35. Griffith, M.E.; Yun, H.C.; Horvath, L.L.; Murray, C.K. Minocycline Therapy for Traumatic Wound Infections Caused by the Multidrug-Resistant Acinetobacter Baumannii-Acinetobacter Calcoaceticus Complex. Infect. Dis. Clin. Pract. 2008, 16, 16-19. [CrossRef]

36. Ning, F.; Shen, Y.; Chen, X.; Zhao, X.; Wang, C.; Rong, Y.; Du, W.; Wen, C.; Zhang, G. A Combination Regimen of Meropenem, Cefoperazone-Sulbactam and Minocycline for Extensive Burns with Pan-Drug Resistant Acinetobacter Baumannii Infection. Chin. Med. J. (Engl.) 2014, 127, 1177-1179. [PubMed]

37. Pogue, J.M.; Neelakanta, A.; Mynatt, R.P.; Sharma, S.; Lephart, P.; Kaye, K.S. Carbapenem-Resistance in Gram-Negative Bacilli and Intravenous Minocycline: An Antimicrobial Stewardship Approach at the Detroit Medical Center. Clin. Infect. Dis. 2014, 59, S388-S393. [CrossRef] [PubMed]

38. Shi, Y.; Xu, Y.; Liu, Y.; Du, W.; Rui, X.; Wang, Y. [Cefoperazone-Sulbactam plus Minocycline in the Treatment of Extensively Drug Resistant Acinetobacter Infections]. Zhonghua Yi Xue Za Zhi 2012, 92, 2847-2850. [PubMed]

39. Wang, L.; Zou, L.; Li, H.; Li, T. [Preliminary Analysis on the Treatment of Ventilator-Associated Pneumonia Caused by Pandrug-Resistant Acinetobacter Baumannii]. Zhongguo Yi XueKeXue Yuan Xue Bao. 2014, 36, 185-188. [CrossRef]

40. Wood, G.C.; Hanes, S.D.; Boucher, B.A.; Croce, M.A.; Fabian, T.C. Tetracyclines for Treating Multidrug-Resistant Acinetobacter Baumannii Ventilator-Associated Pneumonia. Intensive Care Med. 2003, 29, 2072-2076. [CrossRef] [PubMed]

41. Vila, A.; Pagella, H.; Amadio, C.; Leiva, A. Acinetobacter Prosthetic Joint Infection Treated with Debridement and High-Dose Tigecycline. Infect. Chemother. 2016, 48, 324. [CrossRef] [PubMed] 
42. Vay, C.; Nastro, M.; Famiglietti, A.; Rodríguez, C.H. In Vitro Activity of Minocycline Alone or in Combination in Multidrug-Resistant Acinetobacter Baumannii Isolates. J. Med. Microbiol. 2015, 64, 1196-1200. [CrossRef]

43. Yang, Y.-S.; Lee, Y.; Tseng, K.-C.; Huang, W.-C.; Chuang, M.-F.; Kuo, S.-C.; Lauderdale, T.-L.Y.; Chen, T.-L. In Vivo and In Vitro Efficacy of Minocycline-Based Combination Therapy for Minocycline-Resistant Acinetobacter Baumannii. Antimicrob. Agents Chemother. 2016, 60, 4047-4054. [CrossRef] [PubMed]

44. Pei, G.; Mao, Y.; Sun, Y. In Vitro Activity of Minocycline Alone and in Combination with Cefoperazone-Sulbactam Against Carbapenem-Resistant Acinetobacter Baumannii. Microb. Drug Resist. 2012, 18, 574-577. [CrossRef] [PubMed]

45. Liang, W.; Liu, X.-F.; Huang, J.; Zhu, D.-M.; Li, J.; Zhang, J. Activities of Colistin- and Minocycline-Based Combinations against Extensive Drug Resistant Acinetobacter Baumannii Isolates from Intensive Care Unit Patients. BMC Infect. Dis. 2011, 11, 109. [CrossRef] [PubMed]

46. He, S.; He, H.; Chen, Y.; Chen, Y.; Wang, W.; Yu, D. In Vitro and in Vivo Analysis of Antimicrobial Agents Alone and in Combination against Multi-Drug Resistant Acinetobacter Baumannii. Front. Microbiol. 2015, 6, 507. [CrossRef] [PubMed]

47. Bowers, D.R.; Cao, H.; Zhou, J.; Ledesma, K.R.; Sun, D.; Lomovskaya, O.; Tam, V.H. Assessment of Minocycline and Polymyxin B Combination against Acinetobacter Baumannii. Antimicrob. Agents Chemother. 2015, 59, 2720-2725. [CrossRef] [PubMed]

48. Kmeid, J.G.; Youssef, M.M.; Kanafani, Z.A.; Kanj, S.S. Combination Therapy for Gram-Negative Bacteria: What Is the Evidence? Expert Rev. Anti. Infect. Ther. 2013, 11, 1355-1362. [CrossRef] [PubMed]

49. Paul, M.; Carmeli, Y.; Durante-Mangoni, E.; Mouton, J.W.; Tacconelli, E.; Theuretzbacher, U.; Mussini, C.; Leibovici, L. Combination Therapy for Carbapenem-Resistant Gram-Negative Bacteria. J. Antimicrob. Chemother. 2014, 69, 2305-2309. [CrossRef] [PubMed]

50. Poulikakos, P.; Tansarli, G.S.; Falagas, M.E. Combination Antibiotic Treatment versus Monotherapy for Multidrug-Resistant, Extensively Drug-Resistant, and Pandrug-Resistant Acinetobacter Infections: A Systematic Review. Eur. J. Clin. Microbiol. Infect. Dis. 2014, 33, 1675-1685. [CrossRef] [PubMed]

51. Schechner, V.; Kotlovsky, T.; Kazma, M.; Mishali, H.; Schwartz, D.; Navon-Venezia, S.; Schwaber, M.J.; Carmeli, Y. Asymptomatic Rectal Carriage of BlaKPC Producing Carbapenem-Resistant Enterobacteriaceae: Who Is Prone to Become Clinically Infected? Clin. Microbiol. Infect. 2013, 19, 451-456. [CrossRef] [PubMed]

52. The State of the World's Antibiotics, 2015-Center for Disease Dynamics, Economics \& Policy (CDDEP). Available online: https://cddep.org/publications/state_worlds_antibiotics_2015/(accessed on 20 March 2019).

53. Lu, Q.; Luo, R.; Bodin, L.; Yang, J.; Zahr, N.; Aubry, A.; Golmard, J.-L.; Rouby, J.-J. Nebulized Antibiotics Study Group. Efficacy of High-Dose Nebulized Colistin in Ventilator-Associated Pneumonia Caused by Multidrug-Resistant Pseudomonas Aeruginosa and Acinetobacter Baumannii. Anesthesiology 2012, 117, 1335-1347. [CrossRef] [PubMed]

54. Kallel, H.; Hergafi, L.; Bahloul, M.; Hakim, A.; Dammak, H.; Chelly, H.; Hamida, C.B.; Chaari, A.; Rekik, N.; Bouaziz, M. Safety and Efficacy of Colistin Compared with Imipenem in the Treatmentof Ventilator-Associated Pneumonia: AMatched Case-Control Study. Intensive Care Med. 2007, 33, 1162-1167. [CrossRef] [PubMed]

55. Poulakou, G.; Kontopidou, F.V.; Paramythiotou, E.; Kompoti, M.; Katsiari, M.; Mainas, E.; Nicolaou, C.; Yphantis, D.; Antoniadou, A.; Trikka-Graphakos, E.; et al. Tigecycline in the Treatment of Infections from Multi-Drug Resistant Gram-Negative Pathogens. J. Infect. 2009, 58, 273-284. [CrossRef] [PubMed]

56. Shen, F.; Han, Q.; Xie, D.; Fang, M.; Zeng, H.; Deng, Y. Efficacy and Safety of Tigecycline for the Treatment of Severe Infectious Diseases: An Updated Meta-Analysis of RCTs. Int. J. Infect. Dis. 2015, 39, 25-33. [CrossRef] [PubMed]

57. Poulakou, G.; Bassetti, M.; Tsiodras, S. "Excess Mortality" and Colistin-Tigecycline for Extensively Drug-Resistant Acinetobacter Baumannii Bacteremia. Crit. Care Med. 2015, 43, e470-e471. [CrossRef] [PubMed]

58. Liu, Y.-Y.; Wang, Y.; Walsh, T.R.; Yi, L.-X.; Zhang, R.; Spencer, J.; Doi, Y.; Tian, G.; Dong, B.; Huang, X.; et al. Emergence of Plasmid-Mediated Colistin Resistance Mechanism MCR-1 in Animals and Human Beings in China: A Microbiological and Molecular Biological Study. Lancet Infect. Dis. 2016, 16, 161-168. [CrossRef]

59. Tasina, E.; Haidich, A.-B.; Kokkali, S.; Arvanitidou, M. Efficacy and Safety of Tigecycline for the Treatment of Infectious Diseases: A Meta-Analysis. Lancet Infect. Dis. 2011, 11, 834-844. [CrossRef]

60. Yahav, D.; Lador, A.; Paul, M.; Leibovici, L. Efficacy and Safety of Tigecycline: A Systematic Review and Meta-Analysis. J. Antimicrob. Chemother. 2011, 66, 1963-1971. [CrossRef] [PubMed] 
61. Cai, Y.; Wang, R.; Liang, B.; Bai, N.; Liu, Y. Systematic Review and Meta-Analysis of the Effectiveness and Safety of Tigecycline for Treatment of Infectious Disease. Antimicrob. Agents Chemother. 2011, 55, 1162-1172. [CrossRef]

62. Curcio, D.; Verde, P.E. Comment on: Efficacy and Safety of Tigecycline: A Systematic Review and Meta-Analysis Downloaded From. J Antimicrob Chemother J. Antimicrob. Chemother. Adv. Access 2011. [CrossRef]

63. Bassetti, M.; Poulakou, G.; Giamarellou, H. Is There a Future for Tigecycline? Intensive Care Med. 2014, 40, 1039-1045. [CrossRef]

64. Mody, L.; Gibson, K.E.; Horcher, A.; Prenovost, K.; McNamara, S.E.; Foxman, B.; Kaye, K.S.; Bradley, S. Prevalence of and Risk Factors for Multidrug-Resistant Acinetobacter Baumannii Colonization Among High-Risk Nursing Home Residents. Infect. Control Hosp. Epidemiol. 2015, 36, 1155-1162. [CrossRef] [PubMed]

65. Moghnieh, R.; Siblani, L.; Ghadban, D.; El Mchad, H.; Zeineddine, R.; Abdallah, D.; Ziade, F.; Sinno, L.; Kiwan, O.; Kerbaj, F.; et al. Extensively Drug-Resistant Acinetobacter Baumannii in a Lebanese Intensive Care Unit: Risk Factors for Acquisition and Determination of a Colonization Score. J. Hosp. Infect. 2016, 92, 47-53. [CrossRef] [PubMed]

66. Arvaniti, K.; Lathyris, D.; Ruimy, R.; Haidich, A.-B.; Koulourida, V.; Nikolaidis, P.; Matamis, D.; Miyakis, S. The Importance of Colonization Pressure in Multiresistant Acinetobacter Baumannii Acquisition in a Greek Intensive Care Unit. Crit. Care 2012, 16, R102. [CrossRef] [PubMed]

67. Merrer, J.; Santoli, F.; Vecchi, C.A.-D.; Tran, B.; De Jonghe, B.; Outin, H. "Colonization Pressure" and Risk of Acquisition of Methicillin-Resistant Staphylococcus Aureus in a Medical Intensive Care Unit. Infect. Control Hosp. Epidemiol. 2000, 21, 718-723. [CrossRef] [PubMed]

68. Prince, A.S. Biofilms, Antimicrobial Resistance, and Airway Infection. N. Engl. J. Med. 2002, 347, $1110-1111$. [CrossRef] [PubMed]

69. Garnacho-Montero, J.; Dimopoulos, G.; Poulakou, G.; Akova, M.; Cisneros, J.M.; De Waele, J.; Petrosillo, N.; Seifert, H.; Timsit, J.F.; Vila, J.; et al. Task Force on Management and Prevention of Acinetobacter Baumannii Infections in the ICU. Intensive Care Med. 2015, 41, 2057-2075. [CrossRef] [PubMed]

70. Tacconelli, E.; Cataldo, M.A.; Dancer, S.J.; De Angelis, G.; Falcone, M.; Frank, U.; Kahlmeter, G.; Pan, A.; Petrosillo, N.; Rodríguez-Baño, J.; et al. ESCMID Guidelines for the Management of the Infection Control Measures to Reduce Transmission of Multidrug-Resistant Gram-Negative Bacteria in Hospitalized Patients. Clin. Microbiol. Infect. 2014, 20,1-55. [CrossRef]

71. MDRO Management|GuidelinesLibrary|InfectionControl|CDC. Available online: https://www.cdc.gov/ infectioncontrol/guidelines/mdro/index.html (accessed on 19 March 2019).

72. Cheon, S.; Kim, M.-J.; Yun, S.-J.; Moon, J.Y.; Kim, Y.-S. Controlling Endemic Multidrug-Resistant Acinetobacter Baumannii in Intensive Care Units Using Antimicrobial Stewardship and Infection Control. Korean J. Intern. Med. 2016, 31, 367-374. [CrossRef]

73. Chen, C.-H.; Lin, L.-C.; Chang, Y.-J.; Chen, Y.-M.; Chang, C.-Y.; Huang, C.-C. Infection Control Programs and Antibiotic Control Programs to Limit Transmission of Multi-Drug Resistant Acinetobacter Baumannii Infections: Evolution of Old Problems and New Challenges for Institutes. Int. J. Environ. Res. Public Health 2015, 12, 8871-8882. [CrossRef]

74. Enfield, K.B.; Huq, N.N.; Gosseling, M.F.; Low, D.J.; Hazen, K.C.; Toney, D.M.; Slitt, G.; Zapata, H.J.; Cox, H.L.; Lewis, J.D.; et al. Control of Simultaneous Outbreaks of Carbapenemase-Producing Enterobacteriaceae and Extensively Drug-Resistant Acinetobacter Baumannii Infection in an Intensive Care Unit Using Interventions Promoted in the Centers for Disease Control and Prevention 2012 Carbapenemase-Resistant Enterobacteriaceae Toolkit. Infect. Control Hosp. Epidemiol. 2014, 35, 810-817. [CrossRef]

75. Minocycline intravenous-Melinta Therapeutics-AdisInsight. Available online: https://adisinsight.springer. com/drugs/800041651 (accessed on 9 May 2019).

76. Falagas, M.E.; Vardakas, K.Z.; Kapaskelis, A.; Triarides, N.A.; Roussos, N.S. Tetracyclines for Multidrug-Resistant Acinetobacter Baumannii Infections. Int. J. Antimicrob. Agents 2015, 45, 455-460. [CrossRef]

77. Kumar, A.; Ellis, P.; Arabi, Y.; Roberts, D.; Light, B.; Parrillo, J.E.; Dodek, P.; Wood, G.; Kumar, A.; Simon, D.; et al. Initiation of Inappropriate Antimicrobial Therapy Results in a Fivefold Reduction of Survival in Human Septic Shock. Chest 2009, 136, 1237-1248. [CrossRef] [PubMed] 
78. Luna, C.M.; Aruj, P.; Niederman, M.S.; Garzón, J.; Violi, D.; Prignoni, A.; Ríos, F.; Baquero, S.; Gando, S. Grupo Argentino de Estudio de la NeumoníaAsociada al Respirador group. Appropriateness and Delay to Initiate Therapy in Ventilator-Associated Pneumonia. Eur. Respir. J. 2006, 27, 158-164. [CrossRef]

79. Burkhardt, O.; Rauch, K.; Kaever, V.; Hadem, J.; Kielstein, J.T.; Welte, T. Tigecycline Possibly Underdosed for the Treatment of Pneumonia: A Pharmacokinetic Viewpoint. Int. J. Antimicrob. Agents 2009, 34, 101-102. [CrossRef] [PubMed]

80. Imberti, R.; Cusato, M.; Villani, P.; Carnevale, L.; Iotti, G.A.; Langer, M.; Regazzi, M. Steady-State Pharmacokinetics and BAL Concentration of Colistin in Critically Ill Patients After IV Colistin Methanesulfonate Administration. Chest 2010, 138, 1333-1339. [CrossRef] [PubMed]

81. Dimitriadis, P.; Protonotariou, E.; Varlamis, S.; Poulou, A.; Vasilaki, O.; Metallidis, S.; Tsakris, A.; Malisiovas, N.; Skoura, L.; Pournaras, S. Comparative Evaluation of Minocycline Susceptibility Testing Methods in Carbapenem-Resistant Acinetobacter Baumannii. Int. J. Antimicrob. Agents 2016, 48, 321-323. [CrossRef] [PubMed]

82. Wang, P.; Bowler, S.L.; Kantz, S.F.; Mettus, R.T.; Guo, Y.; McElheny, C.L.; Doi, Y. Comparison of Minocycline Susceptibility Testing Methods for Carbapenem-Resistant Acinetobacter Baumannii. J. Clin. Microbiol. 2016, 54, 2937-2941. [CrossRef] [PubMed]

83. Tsakris, A.; Koumaki, V.; Dokoumetzidis, A. Minocycline Susceptibility Breakpoints for Acinetobacter Baumannii: Do We Need to Re-Evaluate Them? J. Antimicrob. Chemother. 2019, 74, 295-297. [CrossRef]

84. Greig, S.L.; Scott, L.J. Intravenous Minocycline: A Review in Acinetobacter Infections. Drugs 2016, 76, 1467-1476. [CrossRef]

85. Jankowski, C.A.; Balada-Llasat, J.-M.; Raczkowski, M.; Pancholi, P.; Goff, D.A. A Stewardship Approach to Combating Multidrug-Resistant Acinetobacter Baumannii Infections With Minocycline. Infect. Dis. Clin. Pract. 2012, 20, 184-187. [CrossRef]

86. Karageorgopoulos, D.E.; Kelesidis, T.; Kelesidis, I.; Falagas, M.E. Tigecycline for the Treatment of Multidrug-Resistant (Including Carbapenem-Resistant) Acinetobacter Infections: A Review of the Scientific Evidence. J. Antimicrob. Chemother. 2008, 62, 45-55. [CrossRef] [PubMed]

87. Ni, W.; Han, Y.; Zhao, J.; Wei, C.; Cui, J.; Wang, R.; Liu, Y. Tigecycline Treatment Experience against Multidrug-Resistant Acinetobacter Baumannii Infections: A Systematic Review and Meta-Analysis. Int. J. Antimicrob. Agents 2016, 47, 107-116. [CrossRef] [PubMed]

88. Zhang, H.; Zhang, Q. Clinical Efficacy and Safety of Colistin Treatment in Patients with Pulmonary Infection Caused by Pseudomonas Aeruginosa or Acinetobacter Baumannii: A Meta-Analysis. Arch. Med. Sci. 2015, 11, 34-42. [CrossRef] [PubMed]

89. Chu, H.; Zhao, L.; Wang, M.; Liu, Y.; Gui, T.; Zhang, J. Sulbactam-Based Therapy for Acinetobacter Baumannii Infection: A Systematic Review and Meta-Analysis. Braz. J. Infect. Dis. 2013, 17, 389-394. [CrossRef] [PubMed]

90. Ozger, H.S.; Cuhadar, T.; Yildiz, S.S.; DemirbasGulmez, Z.; Dizbay, M.; GuzelTunccan, O.; Kalkanci, A.; Simsek, H.; Unaldi, O. In Vitro Activity of Eravacycline in Combination with Colistin against Carbapenem-Resistant A. Baumannii Isolates. J. Antibiot. (Tokyo) 2019, 1. [CrossRef] 